\title{
Molecular line emission from turbulent clouds
}

\author{
V. Ossenkopf ${ }^{\star}$ \\ I. Physikalisches Institut der Universität zu Köln, Zülpicher Straße 77, 50937 Köln, Germany
}

Received 2 January 2002 / Accepted 28 May 2002

\begin{abstract}
In the last years substantial progress has been made in modelling turbulent clouds and describing their structure by characteristic parameters. The missing link for a systematic comparison between models and observations is the lack of efficient radiative transfer algorithms to generate molecular line maps from the models comparable to the observed maps. A fully selfconsistent solution of the radiative transfer problem is computationally very demanding and hardly suited to evaluate a large set of cloud models with regard to their agreement with observed molecular cloud structures. We introduce a new, computationally efficient code to calculate the line profiles based on two reasonable approximations. It is able to compute the molecular line maps in turbulent cloud models with an accuracy of about $20 \%$ fast enough to be run on large sets of model clouds.

Applying the code to hydrodynamic, and magnetohydrodynamic cloud models we study how their structure would appear in molecular line observations. We show that no single molecular line provides a good measure for the density structure in the models. The $X$ factor, translating the integrated line intensities into column densities, can be approximately constant within a density range covering up to a factor 100 in few transitions but for each line this behaviour breaks down outside of a limited range of densities. Optical depth effects and subthermal excitation result in a significant modification of the distribution of line intensities relative to the column density distribution.

All lower transitions of $\mathrm{CO}$ isotopes only trace gas at low and intermediate densities which is distributed over all scales in molecular clouds. Turbulence models driven on the largest scales reproduce the observed scaling behaviour. Higher CO transitions are only excited in dense cores resulting from shocks or gravitational collapse. The existence of massive dense cores resulting from collapse can only be inferred when comparing observations in different transitions taken with an excellent signal-to-noise ratio or from dedicated high-density tracers.

The line profiles obtained from turbulence models driven on large scales break up into several fragments in contrast to observations of molecular clouds without heavy star-formation which show typically smooth profiles with close-to-Gaussian shape. None of the turbulence simulations provides a good match of all observed properties for this type of clouds. The velocity scaling behaviour of all observations and turbulence models is consistent with the interpretation of a molecular cloud as shockdominated medium. More observational data are needed to provide a reliable conclusion on the degree of intermittency.

As molecular lines fail to reflect the density structure of an interstellar cloud line observations should be combined with dust continuum observations to deduce column densities. On the other hand we need the velocity information contained in line observations to discriminate between different turbulence models.
\end{abstract}

Key words. radiative transfer - ISM: clouds - ISM: structure - radio lines: ISM

\section{Introduction}

Images of molecular clouds are surprising with regard to their complexity, frailty and self-similarity. Until now we have no consistent theoretical description for molecular cloud structures. Simple models used to derive cloud parameters from observed molecular lines assume homogeneous structures, simple geometries or the picture of identical non-penetrating clumps in a homogeneous interclump medium. Obviously, they do not provide an adequate description of most aspects of the cloud structure.

On the other hand there are many efforts to set up realistic simulations of hydrodynamic and magnetohydrodynamic turbulence intended to resemble the behaviour of molecular

\footnotetext{
* e-mail: ossk@ph1.uni-koeln.de
}

clouds. But systematic comparisons between molecular cloud observations and turbulence simulations covering several structural properties and a reasonably large set of observations and simulations are still at their very beginning. This is largely due to the complexity of the radiative transfer problem translating the density, temperature, and velocity structure obtained from the turbulence simulations into observable maps of molecular line profiles.

Juvela (1997) provided a self-consistent three-dimensional radiative transfer code for molecular lines based on a MonteCarlo integration scheme. In the pioneering work by Padoan et al. (1998) this code was applied to two magnetohydrodynamic turbulence models. They were able to reproduce several characteristics of observed molecular clouds, like filamentary molecular line images, typical line ratios and line profiles. 
Padoan et al. (2000) analysed the produced line maps to show that an LTE analysis typically underestimates the column densities observed.

Starting from their basic result that the combination of radiative transfer calculations with turbulence models can in principle provide data resembling molecular cloud observations, one can now begin to quantify this resemblance to discriminate between different turbulence models. For this purpose we have to evaluate a large number of models with a reasonable accuracy. Due to computer capacity limitations the computations of Padoan et al. (1998) were still restricted to a spatial resolution of at most $90^{3}$ pixels, a relatively rough angular integration pattern, and the evaluation of two turbulence models only. Mac Low \& Ossenkopf (2000) have shown that for a proper representation of the inertial scaling properties of interstellar turbulence a resolution of 128 pixels in each dimension is essential, 256 pixels are preferable. Although the rapid development of computational technology will allow to treat these resolutions within the Monte-Carlo scheme soon, a quick analysis of a large set of simulations with this resolution is not yet possible in the near future. Thus we use an alternative approach including two basic approximations for the radiative transfer. This approach is not fully self-consistent but allows a fast computation of the observable line data in a reasonable accuracy. We evaluated several hundred models on a standard PC-type computer.

The comparison of the observable molecular line maps from different turbulence simulations with astronomical molecular cloud observations then provides constraints on the mechanisms driving interstellar turbulence and the dynamical conditions in molecular clouds. It can be based on a large variety of statistical measures (see Vázquez-Semadeni 2000; Ossenkopf et al. 2000). We will focus here on the probability distribution functions (PDFs) of the line intensities, the $\Delta$-variance analysis of intensities and velocities, and the shape of the line profiles. We demonstrate how radiative transfer effects change these quantities in different molecular lines.

We have selected a variety of cloud models proposed in the literature to compare their appearance in molecular lines with observational data. We do not aim to fit the behaviour of a particular molecular cloud, but rather discuss general properties. As a kind of reference we will use observations of the Polaris Flare provided by Heithausen \& Thaddeus (1990), Falgarone et al. (1998), and Bensch et al. (2001) as they span a large dynamic range, have a good signal-to-noise ratio and were already analysed with several statistical measures (Falgarone et al. 1998; Heithausen et al. 1998; Ossenkopf \& Mac Low 2002; Bensch et al. 2001). In such a non-starforming region the gas cools very efficiently so that a low uniform temperature is observed. Falgarone et al. (1998) found a gas temperature of about $10 \mathrm{~K}$ throughout the Polaris Flare. On this background we will restrict ourselves to isothermal models. They provide already a large variety of turbulent structures. For active star-forming regions a self-consistent treatment of the energy-balance is essential leading to an additional complication which we want to omit in this first approach.

In Sect. 2 we summarise the cloud models used here. The radiative transfer approximations are introduced and their justification is demonstrated in Sect. 3. In Sect. 4 we discuss the influence of radiative transfer effects on the measured line intensities and the correlation between the cloud density structure and molecular line maps. Section 5 shows how the turbulent velocity structure is represented in the observable line profiles. In Sect. 6 we compare the different cloud models and discuss which models provide reasonable approaches for a detailed representation of observed molecular cloud structures. Section 7 summarises the influence of radiative transfer effects providing guidelines for the observation and analysis of turbulent cloud structures in molecular lines.

\section{Cloud models}

\subsection{Turbulence simulations}

We use existing simulations of hydrodynamic and magnetohydrodynamic turbulence presented by Mac Low (1999), Klessen et al. (2000), Heitsch et al. (2001) and Ossenkopf et al. (2001b). The models are selected to cover different physical scenarios and numerical methods. They are summarised in Table 1 .

The simulations use either a smooth-particle hydrodynamics (SPH) code introduced by Klessen (1997) or the grid-based three-dimensional ZEUS code discussed in detail by Mac Low (1999). These two approaches bracket the true physical behaviour of interstellar turbulence as compressed structures are somewhat too small and rigid in the SPH model and too extended and unstable in the grid-based simulations (Klessen et al. 2000). The SPH computations used here contain $2 \times 10^{5}$ particles. The ZEUS computations are performed on a uniform grid with either $128^{3}$ or $256^{3}$ cells. Although the treatment of a $256^{3}$ grid would have been technically possible we resample all data onto a common $128^{3}$ grid for the radiative transfer computations so that resolution effects do not influence the comparison of the models.

The codes assume periodic boundary conditions. The turbulence in the models is continuously replenished by Gaussian velocity fluctuations with a certain range of wavenumbers as described by Mac Low (1999).

Some models ignore the gravitational interaction as they are designed to represent the behaviour of thin gas on small scales where the free-fall time is large compared to the time of the simulation. Here, the models reach stationary turbulence so that only a single time step is analysed. This stationary turbulence is also used as initial step in models with self-gravity showing the collapse of dense regions. In these models we study several time steps to follow the evolution. We concentrate on models driven on large scales, i.e. with driving wavenumber $k_{\mathrm{d}}=1 \ldots 2$, as Mac Low \& Ossenkopf (2000) have shown that their density structure is consistent with the intensity structure detected in many observations. We add two models driven on small scales (S02 and H02 from Ossenkopf et al. 2001b) to check this conclusion. The models driven on large scales partially include magnetic fields and different strengths of the turbulent driving. This set of simulations covers a wide range of turbulent structures which are physically reasonable for molecular clouds. 
Table 1. Parameters of the considered turbulence models.

\begin{tabular}{llccl}
\hline \hline model name $^{a}$ & numerical method & $k_{\mathrm{d}}{ }^{b}$ & $\mathcal{M}_{\mathrm{rms}}{ }^{c}$ & description \\
\hline S01 in OKH, $\mathcal{B} 1 h$ in KHM & SPH $\left(2 \times 10^{5}\right)$ & $1 \ldots 2$ & 10 & self-gravitating HD turbulence \\
S02 in OKH & SPH $\left(2 \times 10^{5}\right)$ & $7 \ldots 8$ & 10 & self-gravitating HD turbulence \\
H01 in OKH, D1 $h$ in KHM & ZEUS $\left(256^{3}\right)$ & $1 \ldots 2$ & 10 & self-gravitating HD turbulence \\
H02 in OKH, D3h in KHM & ZEUS $\left(256^{3}\right)$ & $7 \ldots 8$ & 10 & self-gravitating HD turbulence \\
M01 in OKH, $\mathcal{E} 1 i$ in HMK & ZEUS $\left(256^{3}\right)$ & $1 \ldots 2$ & 10 & MHD turbulence with $\beta=8 \pi c_{\mathrm{s}}^{2} \rho / B^{2}=0.04$ \\
HC2 in ML & ZEUS $\left(128^{3}\right)$ & $1 \ldots 2$ & 5 & HD turbulence \\
HE2 in ML & ZEUS $\left(128^{3}\right)$ & $1 \ldots 2$ & 15 & HD turbulence \\
\hline
\end{tabular}

\footnotetext{
${ }^{a}$ Names from the papers - ML: Mac Low (1999), KHM: Klessen et al. (2000), HMK: Heitsch et al. (2001), OKH: Ossenkopf et al. (2001b).

${ }^{b}$ Wavenumber of the turbulent driving.

${ }^{c}$ Mach number $\mathcal{M}_{\mathrm{rms}}=v_{\mathrm{rms}} / c_{\mathrm{s}}$ produced by the turbulent driving.
}

\subsection{Scaling}

The turbulence simulations are internally scale free. The scaling of the turbulence simulations to physical quantities was described in detail by Klessen et al. (2000). As we are not interested in the physical timescales here, we need to consider only their relation between the average density $\langle n\rangle$, the size of the cube $L$, and the sound speed $c_{\mathrm{s}}$ (Eq. (9) of Klessen et al. 2000). In an isothermal medium with an effective polytropic index close to unity the sound speed is related to the kinetic temperature $T_{\text {kin }}$ by $c_{\mathrm{s}}=\sqrt{k T_{\text {kin }} / \mu}$ and we obtain

$\left(\frac{L}{0.83 \mathrm{pc}}\right)=\left(\frac{T_{\text {kin }}}{10 \mathrm{~K}}\right)^{1 / 2}\left(\frac{n}{10^{4} \mathrm{~cm}^{-3}}\right)^{-1 / 2}$

adopting an average molecular mass $\mu=2.36 m_{\mathrm{H}}$.

The absolute scaling of the turbulence simulations was chosen to reproduce the conditions in the Polaris Flare at a scale corresponding to the maps taken with the KOSMA $3 \mathrm{~m}$ telescope. Thus we assume a temperature of $10 \mathrm{~K}$ and a total cube size of $5 \mathrm{pc}$, corresponding to $1.9^{\circ}$ at a distance of $150 \mathrm{pc}$ (Bensch et al. 2001). Then the projected cube can be observed in a fully sampled map of $128 \times 128$ points with a spatial resolution of $107^{\prime \prime}$ - the KOSMA resolution in the CO 2-1 transition. From Eq. (1) we obtain an average density of $275 \mathrm{~cm}^{-3}$ resulting in $2000 M_{\odot}$ within the simulated data cube. This is about one third of the total observed mass of the Polaris Flare (Heithausen \& Thaddeus 1990) which is extended over a region of about $7^{\circ} \times 10^{\circ}$. Hence, we have to be aware that the turbulence simulations with the scaling relation by Klessen et al. (2000) may represent only the densest parts of the Polaris Flare where even ${ }^{13} \mathrm{CO}$ starts to become optically thick in the lower transitions (Falgarone et al. 1998).

As reference molecular line we chose the ${ }^{13} \mathrm{CO} 2-1$ transition assuming a molecular abundance $\left[{ }^{13} \mathrm{CO}\right] /\left[\mathrm{H}_{2}\right]=1.5 \times$ $10^{-6}$. To study the effect of different optical depths we change the molecular abundance relative to this "standard" value. The results for $[\mathrm{CO}] /\left[\mathrm{H}_{2}\right]$ around $10^{-4}$ and $2 \times 10^{-7}$ then correspond to ${ }^{12} \mathrm{CO}$ and $\mathrm{C}^{18} \mathrm{O}$, respectively, when we ignore the slightly different frequencies and masses of these isotopes. The molecular constants of the ${ }^{13} \mathrm{CO}$ transitions are taken from the Cologne Database for Molecular Spectroscopy (Müller et al. 2001). We use the collision rate coefficients from Flower \& Launay (1985) assuming a distribution of ortho- and para- $\mathrm{H}_{2}$ in thermal equilibrium (Le Bourlot 1991).

To study how the molecular line behaviour depends on the hydrogen density we keep the total size of the cube constant and vary only the density, then violating the scaling relation by Klessen et al. (2000). A varying molecular excitation by collisions is also traced by considering different transitions. As the ${ }^{13} \mathrm{CO} J \rightarrow J-1$ transitions have increasing critical densities with increasing $J$ we expect similar changes in the molecular lines when going to lower hydrogen densities and when going to higher transition numbers $J$. Although any telescope would observe different transitions with different spatial resolutions we keep the telescope resolution constant here to clearly separate the effect of collisional excitation from other effects.

\section{Radiative transfer in a two-scale approximation}

\subsection{Basic approach}

Ground based observations of molecular lines in the $\mathrm{mm}$ and sub-mm wavelength range face calibration errors resulting from uncertainties in the determination of the beam efficiency, the receiver side-band ratio, and the atmospheric transmission. Moreover, drifts in the system and the atmosphere lead to a temporal variation of the response and calibration parameters of the telescope. Thus an absolute calibration error of about $20 \%$ and relative calibration variations within large maps in the order of $10 \%$ are rather typical. Therefore, it is rarely necessary to use radiative transfer codes which are accurate down to the percent level as they are available for one-dimensional problems (see e.g. Ossenkopf et al. 2001a). Here we introduce an approximative approach, especially suited for turbulence simulations, which is accurate to about $20 \%$ for the considered models. It is thus adequate for comparisons to typical molecular line observations.

The accuracy of the approximations was computed for each model listed in Sect. 2.1 by the estimators discussed below. In the following we introduce the different parts of the radiative transfer code using the model $B 1 h$ from Klessen et al. (2000) (model S01 from Ossenkopf et al. 2001b) for a demonstration. This is a hydrodynamic model driven on large scales including gravitational collapse. We will see below that it represents a 
"worst case" scenario with respect to the approximations in the radiative transfer because of relatively low Mach numbers and a dominant large-scale structure. Results for the other models will be mentioned only if they change some of the conclusions relative to this model which we will call "reference" model in the following. If the radiative transfer code is to be applied to molecular cloud simulations using different approaches than the models in Sect. 2.1 the tests for the accuracy of the approximations should be repeated in the same way because there is no general analytic expression for the errors of the approximations holding in arbitrary configurations.

For the discussion of the general line radiative transfer problem we refer to a standard textbook (e.g. Mihalas 1978). The crucial quantity in the radiative transfer problem is the radiative energy density in a given point $\boldsymbol{r}$ which can be absorbed by the local material. This quantity enters the balance equations needed to compute the molecular level populations. For each transition $i$ it is given by

$u_{i}(\boldsymbol{r})=\frac{1}{c} \int_{4 \pi} \mathrm{d} \Omega \int_{-\infty}^{\infty} \mathrm{d} v I_{i, v}(\boldsymbol{r}, \boldsymbol{n}) \Phi_{i, v}(\boldsymbol{r}, \boldsymbol{n})$.

Here, $I_{i, v}(\boldsymbol{r}, \boldsymbol{n})$ is the intensity in the transition $i$ at frequency $v$ from the direction $\boldsymbol{n}$ and $\Phi_{i, v}(\boldsymbol{r}, \boldsymbol{n})$ is the local absorption profile. In a self-consistent solution of the problem, the intensity $I_{i, v}$ is computed for each direction by integrating the radiative transfer equation through the whole cloud up to point $\boldsymbol{r}$. Here, we introduce approximations for $u_{i}$ which do not require to compute the intensities coming from all directions at all points. We can exploit the fact that the integration over all angles $\mathrm{d} \Omega$ and over all frequencies $\mathrm{d} v$ cancels out many fluctuations in the spatial distribution of the radiation field and in the exciting line profiles.

\subsection{The local large-velocity gradient approximation}

Turbulent clouds are dominated by supersonic shocks running through the interstellar medium (Ballesteros-Paredes et al. 1999b). They create large velocity gradients in most parts of a molecular cloud. Hence, the velocity component along any line of sight changes by more than the thermal line width on a short distance. Remote points cannot radiatively excite each other because their Doppler shifted emission and absorption profiles do not overlap, except for few distant points where the lineof-sight velocity coincides with a certain probability. The restriction of the local radiative interaction to short length scales can be exploited in the radiative transfer computations. This is known as the large-velocity gradient (LVG) approximation introduced by Sobolev (1957).

To check the assumption of large velocity gradients in the cloud models we have computed for each pixel in the simulated data cubes the radiative interaction lengths given by the local velocity gradients in the different directions. The interaction length is determined by

$L_{\sigma}=\left|\frac{\sigma}{\mathrm{d} v_{n} / \mathrm{d} r}\right|$,

when the local velocity distribution is $\Phi(v)=$ $1 / \sqrt{\pi \sigma^{2}} \exp \left(v^{2} / \sigma^{2}\right)$ and $v_{n}$ is the velocity component

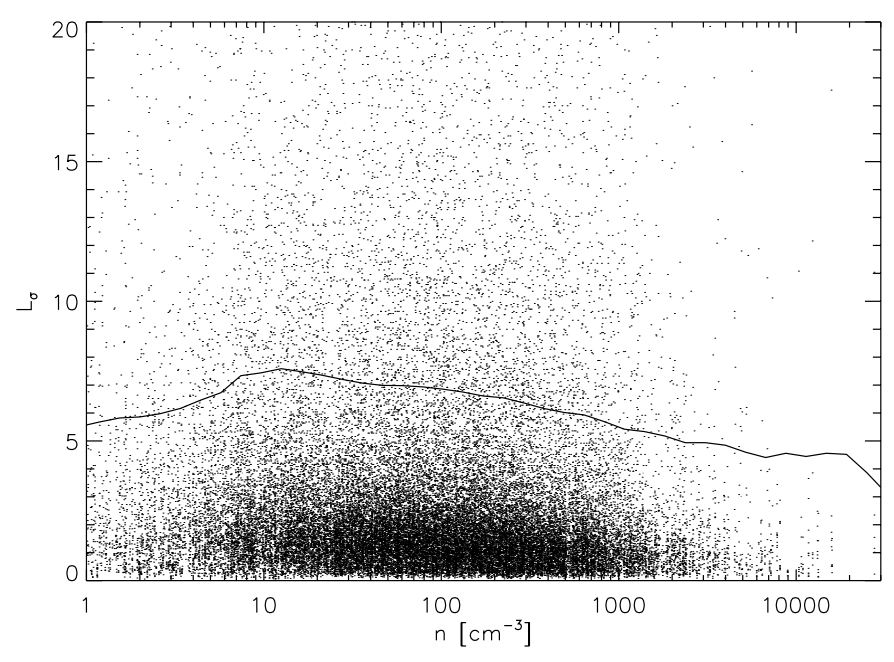

Fig. 1. Radiative interaction lengths (in pixels) computed from the velocity gradients in the six orthogonal grid directions. The dots show the values for 8000 pixels in the model cube. The solid line is the $90 \%$ probability limit in the distribution of all pixels in the cube falling into different density bins.

in the considered direction (Ossenkopf 1997). For ${ }^{13} \mathrm{CO}$ at $10 \mathrm{~K}$ the thermal width is $\sigma=75 \mathrm{~m} \mathrm{~s}^{-1}$. Using the velocity variation between two neighbouring pixels $\Delta v_{i}$ for the gradient we obtain the interaction length $L_{\sigma}=\left|\sigma / \Delta v_{i}\right|$ in units of the pixel size.

In Fig. 1 we have plotted the six orthogonal interaction lengths of 8000 sparsely distributed pixels as a function of their density in the reference turbulence model before the onset of gravitational collapse. We have also plotted the $90 \%$ probability limit of the full distribution as a solid line. The distribution shows only a weak density dependence. The main influence of the density is the different number of pixels at each density, so that no separate discussion of different densities is required. For some pixels we find interactions lengths outside of the plotted range. Few of them even show a value above 1000 due to a very small velocity difference in a certain direction. However, for $90 \%$ of the pixels the interaction length is below about 6 . Mac Low \& Ossenkopf (2000) have found that numerical dissipation blurs structure below about 10 pixels in the simulations. The radiative interaction length just falls into this range, i.e. only pixels within the numerical smoothing length are directly coupled radiatively.

The LVG approximation now assumes that all gas parameters are constant within the interaction length so that the excitation can be computed from local gas parameters and the size of the interaction region only. Although for most pixels the interaction length falls below the dissipation length of the simulations one can expect deviations from the assumption of constant parameters. We have computed the relative density variation $\Delta n / n$ within the interaction length for the different models and found that the assumption of a zero gradient is not justified, but for most pixels the density does not change by more than a factor 2 within the interaction length. Ossenkopf (1997) showed that this density variation can be treated by a linear extension of the LVG approximation where the radiative interaction is computed not only from the local 


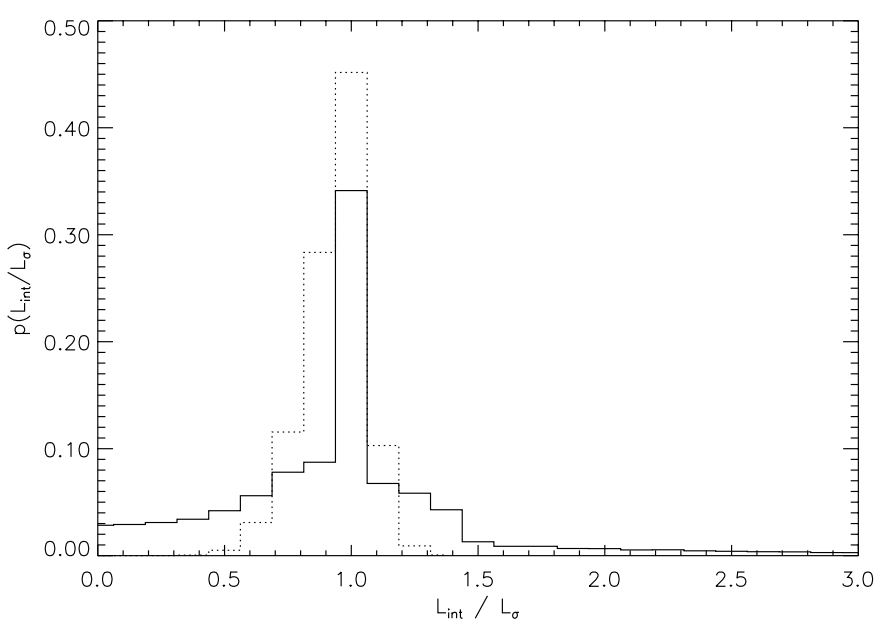

Fig. 2. Distribution of the ratio between the interaction length measured as the number of pixels needed to change the velocity by $\sigma$ and the interaction length computed from the local velocity gradient in each direction (solid line). The dotted histogram shows the same distribution when computed for the geometric mean of the six orthogonal directions for each pixel.

gas properties but also from the local gradients. It was demonstrated that the line intensities computed in this way agree within $20 \%$ with the results from the ordinary LVG approximation if the absolute density variation within the interaction region is below 1 . Because the use of the linear extension is numerically simple and does not require additional computing power, we use this method to treat density variations within the interaction region in the radiative transfer code, even if the error that would be made by the ordinary LVG approximation it is not very large.

Another error can be made by the extended LVG approximation when the interaction length computed from the local velocity gradient by Eq. (3) is not identical to the true size of the interaction region. To check this condition we count the number of pixels which are actually needed to change the lineof-sight velocity by the local line width $\sigma$ in the six grid directions from each point. Figure 2 shows the distribution of the ratio between this length $L_{\text {int }}$ and the interaction length $L_{\sigma}$ from Eq. (3). The small imbalance of the distribution towards values below one results from the discrete counting. We see that for most pixels and directions the velocity gradient is a very good measure for the true interaction length, but that there are still many cases with a considerable deviation. However, the radiative energy is determined only by the angular average, which will cancel out many of the length fluctuations. Positive and negative differences between $L_{\text {int }}$ and $L_{\sigma}$ in different directions reduce the total uncertainty of the size of the interaction region. As the interaction length does not enter the radiative energy linearly we cannot give a general formula for the reduction of the error by angular integration. The quantity entering linearly into the radiative energy density is the escape probability which is close to zero for very long and close to one for very short interaction lengths. To obtain a reasonable guess for the reduction of the uncertainty by angular integration we have added in Fig. 2 the distribution of the geometric angular average of $L_{\text {int }} / L_{\sigma}$ for each pixel. For most pixels the two lengths agree within $20 \%$ but we still find a considerable number of pixels with deviations up to $40 \%$. Detailed parameter studies for the LVG approximation by White (1977) and Snell (1981) showed that a change of the column density, i.e. the interaction length, by a factor two will change the line intensities by at most a factor 1.6, but typically by much lower values. For single pixels we thus obtain maximum deviations up to about $25 \%$ typically resulting in errors below $10 \%$ when integrating the radiation from several pixels along the line of sight.

The six directions considered here for each pixel cannot reflect the exact shape of the interaction region. As the typical interaction length is small and the structure relatively smooth throughout the interaction region, it is however justified to assume a smooth angular variation of the interaction length, so that the interaction region is well represented by the values in the six grid directions. Moreover the angular integration of the radiation field entering the excitation problem reduces the influence of uncertainties of the interaction length in any particular direction.

Altogether, the vast majority of the model pixels and especially the dense pixels providing the main contribution to the molecular line emission have a small local radiative interaction region so that an LVG approach is justified. Although the velocity gradient and the density are not constant within the interaction region their typical variation can be treated with a reasonable accuracy in an extended LVG approximation. We find that the LVG approximation is better in models driven at large Mach numbers because they produce steeper velocity gradients. It is somewhat worse in the small-scale driven models because the velocity changes occur on smaller scales so that the typical difference between $L_{\text {int }}$ and $L_{\sigma}$ is larger by $20-30 \%$ compared to Fig. 2. In general the approximation should be usable as long as the total velocity dispersion is at least 5-10 times the local velocity dispersion and the density structure is not dominated by fluctuations within the radiative interaction length. Future investigations should test this second condition for other types of turbulence simulations which show less blurring on small scales.

\subsection{The "average isotropic field" approximation}

The extended LVG approximation provides a description for the radiative interaction within the close neighbourhood of each point but it does not include the interaction between distant points with the same line-of-sight velocity. This long range interaction may be inferred from the general structure of turbulent molecular clouds. They are characterised by a strongly irregular behaviour. Many clumps and filaments contribute to the radiation field instead of systematic structures. If we assume that the probability of finding a point emitting with a certain velocity is about the same in each direction and the integration over large angular ranges results in a good sampling of this probability distribution we expect that the line shape averaged over a large spatial angle will be about the same along all lines of sight. The intensity is then mainly determined by the distance from the corresponding edge of the cloud. Because the radiative excitation is governed only by the absorbed radiation 


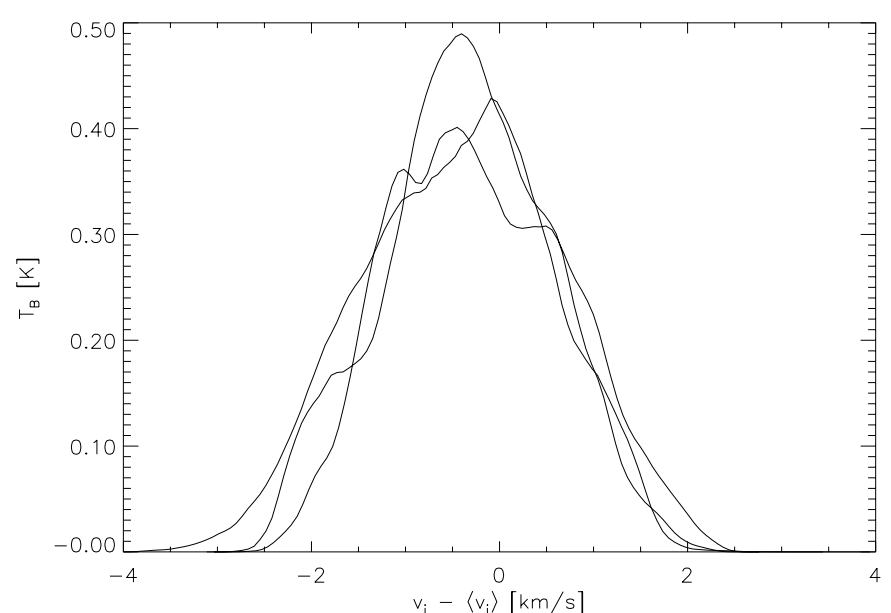

Fig. 3. Average line profiles in the ${ }^{13} \mathrm{CO} 2-1$ transition obtained in three perpendicular directions for the reference turbulence model.

field integrated over all directions it is sufficient to consider this average line shape for the solution of the excitation problem.

We have tested the large scale isotropy of the molecular lines carrying out radiative transfer computations in the different rectangular directions of the model cubes. In this treatment we assume that it is equivalent to consider either one point in the cloud and the angular average over a large range of spatial directions, or a plane in the cloud and the average of all rays falling from one direction onto that plane. The second method has the technical advantage that we only need to compute the radiative transfer along the grid axes. Although both approaches do not produce exactly matching profiles they trace the total volume of the model data cube in a similar way so that they are equivalent in terms of the fluctuations of the radiation field. For the turbulence models driven on small scales we obtain Gaussian line profiles almost identical in all directions. This is due to the large number of independent shock structures so that the statistical approach is completely adequate. The situation is worse in the case of turbulence models dominated by few large scale structures. Figure 3 shows the average line profiles in three directions for the initial state of the reference model with the large-scale driving. The profiles have a similar appearance but clear variations in the exact line shape.

Taking into account that the radiative energy density is integrated over all frequencies (Eq. (2)) and that the absorption profile of most pixels scans a large part of the external radiation profile by its own velocity when looking into different directions, the excitation does not depend sensitively on the exact line profile. The integrated line intensities from Fig. 3 vary by less than $10 \%$.

With a large-scale isotropy of the emission profile, the exciting radiation field depends only on the distance of the point of interest from the cloud edges. Unfortunately, the appropriate definition of molecular cloud edges is still an open issue (Ballesteros-Paredes et al. 1999a). Different observations provide strongly different values for the spatial extent of a molecular cloud when different molecules or transitions are considered. The simulations on their hand simply ignore all edge effects assuming periodic boundary conditions. With this background we stay away from artificially inventing a "proper"

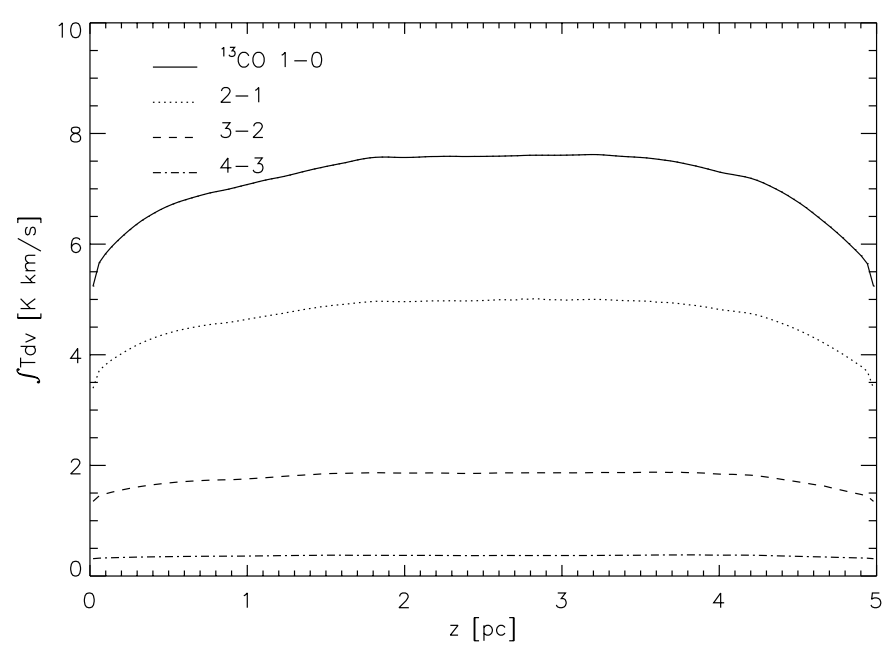

Fig. 4. Average integrated line intensity from positive and negative $z$ direction in ${ }^{13} \mathrm{CO}$ as a function of the distance from the edge of the cloud. To demonstrate a situation with strong variations we have increased here the molecular abundance of ${ }^{13} \mathrm{CO}$ by a factor 50 relative to the standard value.

treatment of cloud edges and follow the concept of the structure simulations treating each part of the cloud as equivalent. We thus use one large scale radiation field for the excitation problem throughout the whole cloud. This average field and finally the observable line profiles depend, however, on the total thickness of the cloud. We made several tests using either only parts of the simulated data cubes or piling up some of them to a larger system but found no new effects compared to the treatment of single cubes, so that we use their size here as the depth of the cloud.

Now we have to identify those points where to compute the representative exciting radiation field. Here, contributions from different directions have to be combined. When replacing the angular average by the average within a plane we can use a one-dimensional picture where the intensity accumulated on the way from one edge up to the plane of interest and the intensity from the opposite edge are added. Figure 4 shows the variation of this average integrated line intensity of the four lowest ${ }^{13} \mathrm{CO}$ transitions in the $z$ direction throughout the data cube. As the variations are very small for the standard model abundances we have artificially enhanced the molecular abundance here by a factor 50 to show a remarkable effect. As long as the lines are optically thin we find a perfect match of the constant field approximation. The lower transitions at the increased abundance, however, show optical depths up to several hundreds in the densest parts of the cloud. We see a significant drop of the average field towards the edges of the cloud and small asymmetries in the curves corresponding to the location of the most prominent density enhancements in the cube. A further increase of the optical depth results in minor changes to the shape of the curve in the lower transitions but extends the curvature also to the higher transitions. For all models the intensity is constant within $10 \%$ throughout the cloud except for the two regions at the outer edges covering each about $5 \%$ of the cube size. Thus we can select any point that is not too close to the edge of the cube to compute the average radiation field. We use an averaging plane which is $1 / 4$ of the cube size 
away from the edge. The figure shows, however, that for optically thick lines the radiative excitation is systematically overestimated at cloud edges. This can lead to an underestimation of self-absorption effects in lines which are optically thick but only weakly excited by collisions. A systematic investigation of this edge effect should be added when models with a selfconsistent edge description become available.

We must admit that the use of this average exciting line profile for the long range radiative interaction neglects all effects which might arise from a large scale anisotropy of the cloud. It also implies an isotropic radiation field illuminating the molecular cloud from the outside, which was assumed to be only the cosmic background radiation here. Moreover, it is heavily based on the assumption of a statistical equivalence of different points in the turbulence model. As the average radiation field always also contains a contribution from the considered pixel - although at some distance - the approximation works only if this contribution can be neglected within the whole radiation field. In the turbulence models the contribution from every single interaction region is insignificant but the same method cannot be used for configurations with only a few peculiar objects. In the turbulent structures the main uncertainty, however, comes from the treatment of the cloud edges, so that a more sophisticated model makes no real sense as long as the turbulence simulations themselves do not include edge effects.

As we have no absolute measure for the error in the line intensities that is produced by this approximation we show in Fig. 5 the relative difference in the ${ }^{13} \mathrm{CO} 2-1$ map when the long range radiative interaction is either taken into account by the average line profile or completely neglected. The error due to an imperfect treatment of the long-range interaction will be a small part of this deviation. The large-scale field produces differences up to $70 \%$ but only in "empty" areas of the map, where the low density gas is excited by global radiation field. In the denser regions of the data cube the excitation at each point is dominated by the local excitation treated within the LVG approximation. Thus the difference is close to zero for those parts of the map providing the main emission. The difference in the total integrated intensity between the to maps is $16 \%$. The effect is lowered both for higher and lower molecular abundances because the mutual excitation is decreased if large parts of the cloud are optically thick or if the dense regions are not sufficiently bright. It is increased in the models with collapsed cores where the total dynamic range of densities is larger than in Fig. 5, but in these cases an extremely good signal-to-noise ratio would be required to detect the emission from the low density regions so that the increased error is hardly detectable. Taking the $10 \%$ typical uncertainty of the large scale radiation field from Fig. 4 and the maximum influence of the large scale field of about $70 \%$ from Fig. 5 we expect an overall uncertainty coming from the approximation of an average largescale field below $10 \%$. We plan a comparison with the fully self-consistent code of Juvela (1997) for a few test cases to get a better estimate of the exact limitations.

Altogether, the local excitation at each point is computed by the combination of two approximations. The radiative interaction with the direct environment is given by the linear
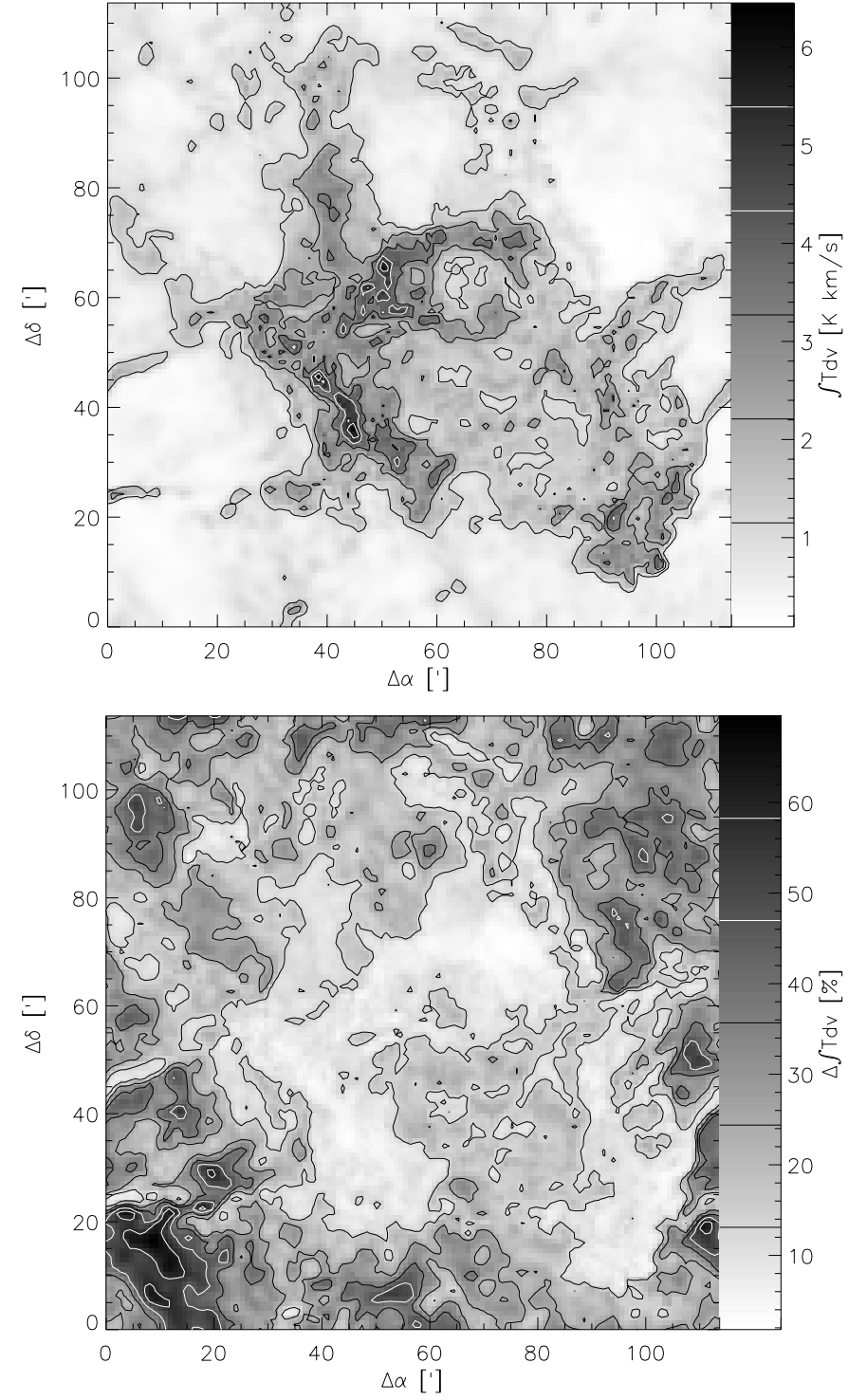

Fig. 5. Comparison of the integrated line maps in ${ }^{13} \mathrm{CO} 2-1$ for the reference model before the onset of gravitational collapse, computed with and without the large-scale radiation field. As it is difficult to see any difference between the two maps by eye we have plotted in the upper plot the map with large-scale radiative excitation and in the lower plot the relative difference between this map and the map obtained without the large-scale field.

extension of the LVG approximation. The interaction with distant points is treated by adding an average, large-scale isotropic field as external field to the local LVG treatment. The average external line profile is computed by a radiative transfer integration through the whole data cube from both sides up to a plane in the interior of the cube. The combination of both steps requires an iterative scheme which is solved by means of an accelerated $\lambda$-iteration for the radiative energy densities (Auer 1987).

\subsection{Collection of similar pixels}

With the two-scale approximation described above one can solve the excitation problem on a $128^{3}$ grid within about 


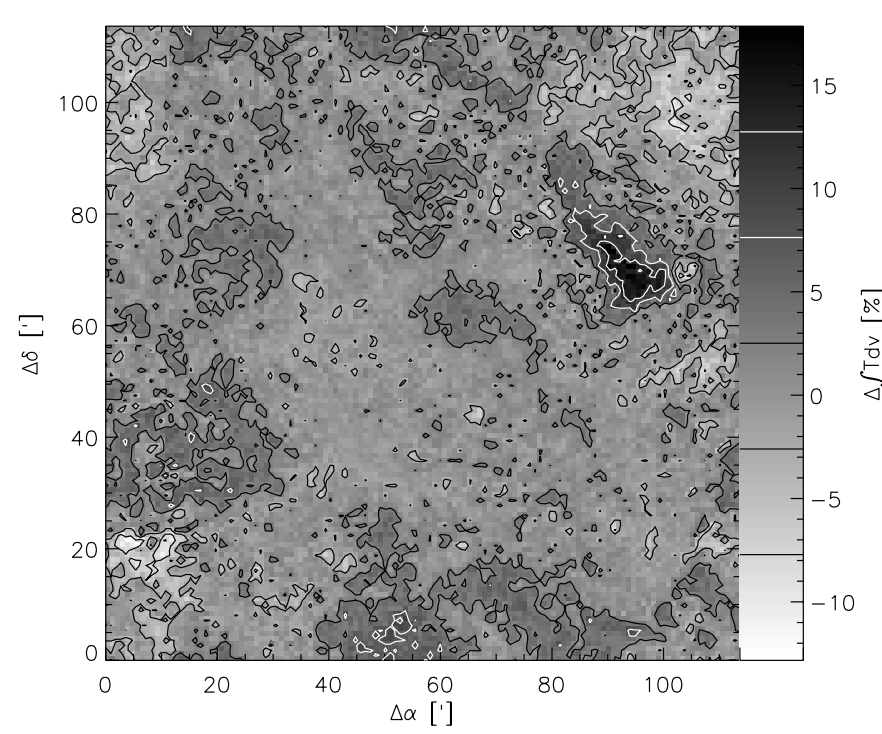

Fig. 6. Relative differences between the line intensities computed by means of a collection of similar pixels into a table and the intensities where the excitation is computed separately for each pixel (shown in Fig. 5).

4 hours on personal computer with 700-MHz-CPU. A further acceleration is possible when the excitation problem is not solved individually for each pixel but pixels with similar properties are collected in a table and the excitation is computed for all table entries. As we assume that the large scale radiation field is constant throughout the cube, the table is spanned by local quantities only. We use a two-dimensional scheme where the pixels are collected corresponding to their density and to their average velocity gradient ${ }^{1}$. As long as the size of the table is lower than about a hundredth of the cube size, the total runtime of the code is dominated by the computation of the average line profiles, so that we get a speedup by about a factor 100 , reducing the total time needed for a cube to a few minutes.

The choice of the table guarantees that the average gradient and the density of each pixel are accurately reflected because they are the dominant quantities in the computation of the excitation. For the other parameters like the dispersion of the velocity gradient in different directions and the density gradient as function of the velocity gradient we take the average from all pixels represented by a table entry. Thus the table entries do not necessarily match the properties of each pixel. Hence, we expect small local variations of the resulting line data compared to the model where each pixel is treated individually. In Fig. 6 we show the relative differences in the integrated map for the reference model. It should be compared to the integrated map in Fig. 5. We find deviations up to about $15 \%$, but both strong positive and negative deviations only in regions of almost zero intensity. In regions of noticeable emission the collection of pixels into the table produces only very small errors. The difference in the integrated map falls below $0.2 \%$. For the small scale driven models all deviations are still somewhat lower.

\footnotetext{
1 With a physical model for the cloud edges, the distance of a point from the cloud edge would enter as third dimension of the table so that the speed-up of the code by the collection of similar pixels would be drastically reduced.
}

We have varied the size of the table between 60 and 400 entries for the density and between 7 and 40 entries for the average velocity gradient to see whether an increase of the table size improves the accuracy of the approach. Whereas an increase up to about $120 \times 15$ leads to a noticeable reduction of the errors, larger tables do not result in a further improvement as long many pixels are represented by one table entry. The two dimensional scheme does not reproduce deviations from a regular interaction region or from the average density gradient in some of the pixels. For a better representation we would need a table size which is no longer small compared to the cube size, so that we accept the error demonstrated above when we need the accelaration. We use both versions of the numerical code for different parts of this study. All tests on the radiative transfer itself are carried out with the slow program version. The systematic computations to dismantle the astrophysically observable relations discussed in Sects. 4-6 were done, however, with the fast version allowing to test several hundred parameter-model variations.

Although we have restricted ourselves here to isothermal models the radiative transfer problem can be solved in the same way for clouds with an internal temperature structure. Then, the local large-velocity gradient approximation also has to take the local temperature gradient into account in the same way as local density gradients are treated here. Unfortunately, the accuracy of the average isotropic field approximation will be further reduced because every additional dimension of the parameter space can lead in principle to stronger local anisotropies. Moreover, the relative reduction of the computing time by a collection of similar pixels in a table will be smaller than in the isothermal case as the table has to be at least three-dimensional. Before applying the radiative transfer code to non-isothermal clouds these aspects should be tested in the same way as performed here, to estimate the reliability of the results. These tests, however, go clearly beyond the scope of this paper so that we have to refer to future work.

\subsection{Line profiles, beam smearing and noise}

When the excitation problem is solved using the two-scaleapproximation discussed above we compute the observable line map in a separate step. The radiative transfer equation is integrated for the selected transition along rays in $z$-direction through the centres of all pixels. All quantities are linearly interpolated between neighbouring grid points in this step. At each point the local absorption and emission profile is taken to follow a thermal Gaussian. Velocities differing by more than $1.5 \mathrm{FWHM}$ from the line centre velocity are neglected. The frequency resolution is assumed to be $63 \mathrm{~m} \mathrm{~s}^{-1}$, which is half the $F W H M$ of the thermal velocity distribution.

The resulting intensities on the grid of rays are convolved by a Gaussian beam of the selected FWHM to compute the final map of observable line profiles. To produce realistic images we may add white noise to the data to obtain data cubes which are directly comparable to observations with a certain signalto-noise ratio. In most figures shown here we omitted the beam convolution and the addition of noise to demonstrate the pure 

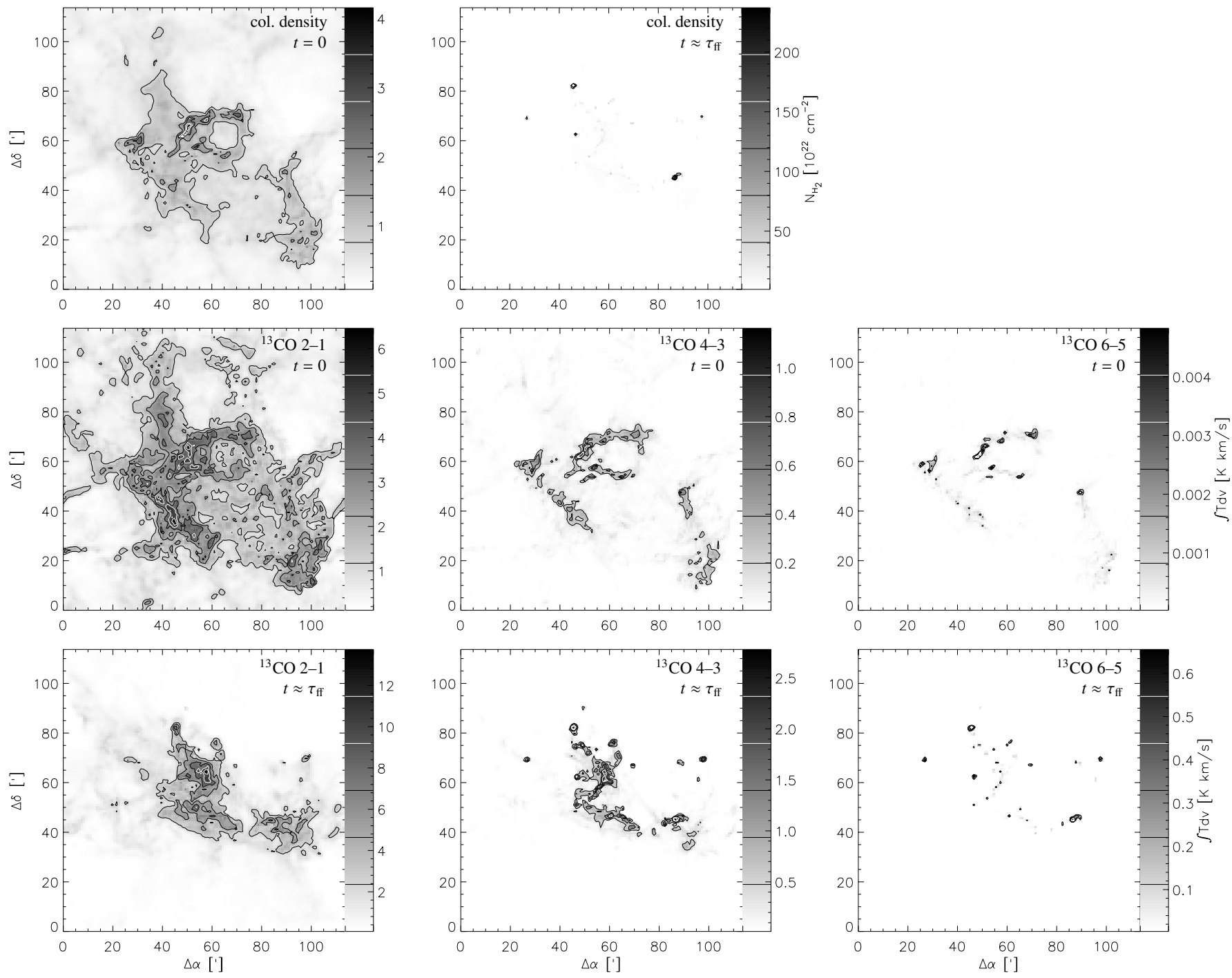

Fig. 7. Column density maps (first row) and resulting ${ }^{13} \mathrm{CO}$ integrated line maps for the $2-1,4-3$, and $6-5$ transition (from left to right) for the reference model at the beginning of the gravitational collapse (second row) and after the gravitational collapse of $30 \%$ of the gas mass into dense cores (third row).

radiative transfer effects. The beam convolution was applied for the line profiles discussed in Sect. 5.1 and both effects are added in the discussion of the scaling behaviour at the end of Sect. 4.4. They are also taken into account when directly comparing the properties of the simulated line maps with observational data.

\section{The resulting intensity structure}

\subsection{Retrieval of characteristic density features from molecular line maps}

In a first glance at the results from the radiative transfer simulations we compare different maps obtained from the large scaledriven model at the beginning of the gravitational collapse and after one free-fall time. In Fig. 7 we show the maps observable in three different ${ }^{13} \mathrm{CO}$ transitions and the underlying column density structure. The corresponding ${ }^{13} \mathrm{CO} 1-0$ and 3-2 maps fit well into the visible trend that with growing transition number $J$ the total intensity decreases and the emission becomes less and less space filling, breaking up smooth structures into different clumps.

The density structure strongly differs between the two time steps of the model. Whereas the densest shocks in the first step fall at gas densities of about $4 \times 10^{4} \mathrm{~cm}^{-3}$ the collapsed cores in the second step show densities which are about one hundred times higher. Thus the column density map is completely dominated by these cores in the second step. In the 2-1 maps we find, however, in both cases extended emission which is only somewhat brighter and spatially stronger confined in the collapsed model. The low- $J$ transition hardly notices the formation of dense cores. The loss of $30 \%$ of the total mass into cores is barely visible.

In the initial step the 3-2 transition, which is not shown in the figure, is the best tracer for the general density structure. In contrast only the 6-5 transition reflects the true density structure of the collapsed model. This knowledge is, however, not very useful as observational guide to search for dense cores in cold molecular clouds as the ${ }^{13} \mathrm{CO} 6-5$ line is weak 
with peak temperatures of about $0.2 \mathrm{~K}$ and peaks away from the peak emission in the 4-3 transition. Thus, one would have to map a large area of a molecular cloud down to low noiselevels to detect the cold cores. Here, dust observations are the better approach to find dense clumps although their interpretation is also not straight forward (Lada et al. 1994).

In corresponding maps computed for ${ }^{12} \mathrm{CO}$ abundances the subthermal excitation by collisions in thin regions is partially compensated by excitation from optically thick radiation. Thus the ${ }^{12} \mathrm{CO} 4-3$ maps are very similar to the ${ }^{13} \mathrm{CO} 2-1$ maps and the ${ }^{12} \mathrm{CO} 6-5$ maps resemble the ${ }^{13} \mathrm{CO} 4-3$ maps although their intensity is somewhat lower. The maps in $\mathrm{C}^{18} \mathrm{O}$ follow the same behaviour having the same appearance as corresponding ${ }^{13} \mathrm{CO}$ maps taken in the next higher transition.

\subsection{Non-LTE excitation, optical depth and the mass conversion factor}

After this phenomenological comparison we enter a detailed discussion to quantify the radiative transfer effects. We will start discussing the widely used $X$ factor translating integrated CO line intensities into column densities: $X=N_{\mathrm{H}_{2}} / I$ with $I=\int T_{\mathrm{B}} \mathrm{d} v$. For several nearby clouds Digel et al. (1996) and Meyerdierks \& Heithausen (1996) derived $X$ factors for the ${ }^{12} \mathrm{CO} 1-0$ intensity between $0.35 \times 10^{20} \mathrm{~cm}^{-2}\left(\mathrm{~K} \mathrm{~km} \mathrm{~s}^{-1}\right)^{-1}$ for the Polaris Flare and $2.5 \times 10^{20} \mathrm{~cm}^{-2}\left(\mathrm{~K} \mathrm{~km} \mathrm{~s}^{-1}\right)^{-1}$ for the Perseus arm assuming the ratio to be constant within the clouds. Meyerdierks \& Heithausen (1996) admitted however that $X$ might vary by about a factor two within one cloud when considering either the translucent or the diffuse parts. The conversion factors for the other transitions are then often rescaled by the typical or average line ratio (e.g. Heithausen et al. 1998).

The true conversion factor is constrained by molecular properties. For LTE excitation the specific emission of each molecule is a constant. In the optically thin case it may be summed up over the molecular column density along the line of sight to the maximum total intensity. Subthermal excitation and optical depths effects can only reduce the emission per column density relative to this LTE value ${ }^{2}$. For CO at $10 \mathrm{~K}$ the corresponding theoretical minimum conversion factors, given in $\mathrm{cm}^{-2}\left(\mathrm{~K} \mathrm{~km} \mathrm{~s}^{-1}\right)^{-1}$, are $9.3 \times 10^{14}$ in $1-0,6.5 \times 10^{14}$ in $2-1$, $1.5 \times 10^{15}$ in $3-2,7.5 \times 10^{15}$ in $4-3$, and $1.5 \times 10^{18}$ in the $6-5$ transition. At a molecular abundance $\left[{ }^{12} \mathrm{CO}\right] /\left[\mathrm{H}_{2}\right]=10^{-4}$ the theoretical 1-0 conversion factor is only one fourth of the lowest $X$ value derived for the Polaris Flare. Thus the observed numbers may indicate that in this cloud about $75 \%$ of the molecular mass are hidden to the observations by subthermal excitation and optical depth effects. The effect is even stronger in the 2-1 transition where the LTE molecular brightness is higher but the typically observed intensities are lower than in ${ }^{12} \mathrm{CO} 1-0$.

\footnotetext{
${ }^{2}$ In a few models assuming very low abundances we found some points in the maps which show suprathermal emission in the 1-0 transition. The low- $J$ transitions can be slightly suprathermally excited in case of high densities and low optical depths. This might occur e.g. in $\mathrm{C}^{18} \mathrm{O}$ observations but is hard to detect due to the weakness of the lines.
}
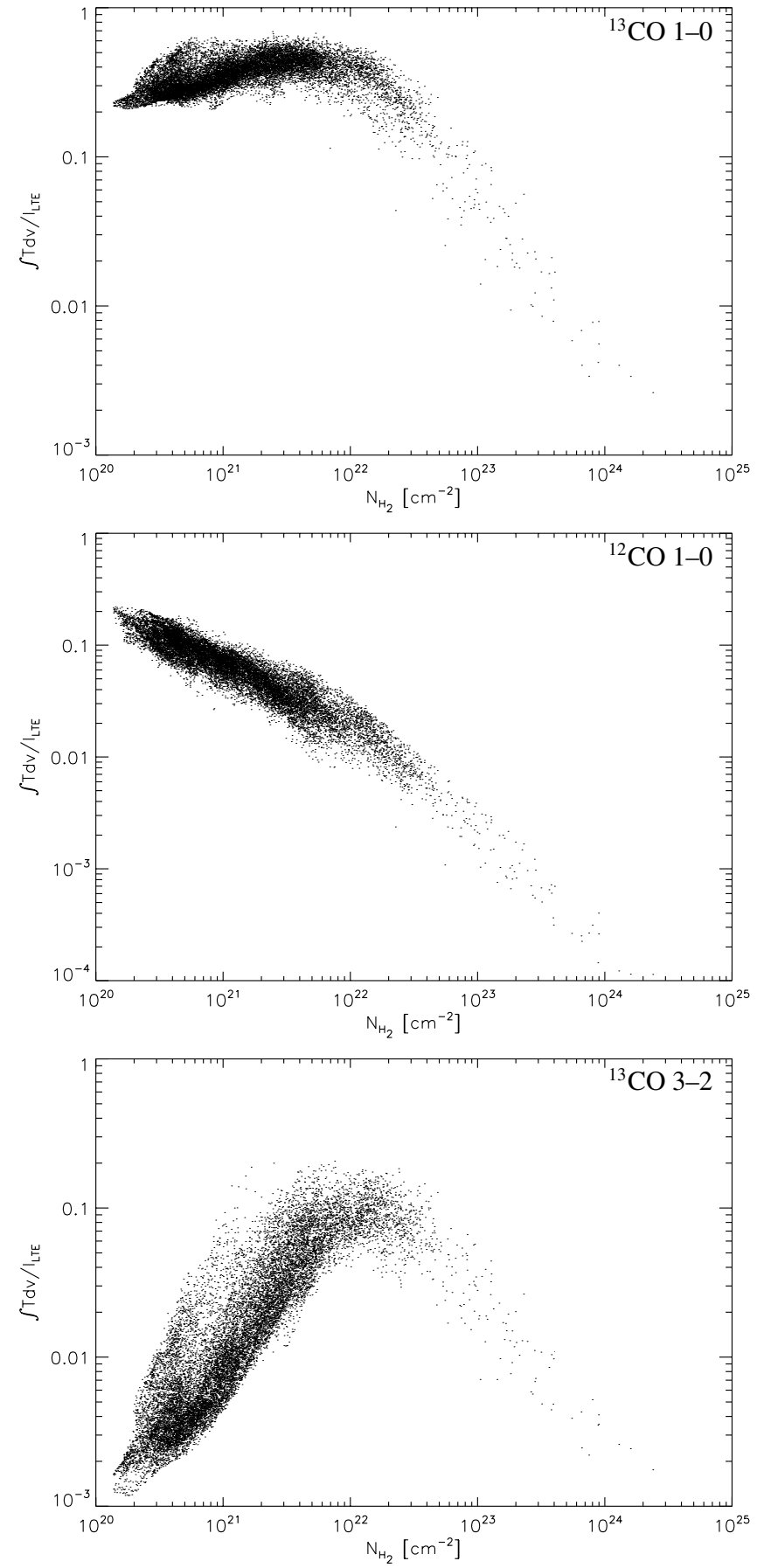

Fig. 8. Line intensities relative to the line-of-sight integrated LTE emissivity as a function of the column density for each point in the maps obtained from the collapsed model. The upper plot shows the ${ }^{13} \mathrm{CO} 1-0$ transition, the central plot is the same transition but for ${ }^{12} \mathrm{CO}$ molecular abundances, the lower plot represents the ${ }^{13} \mathrm{CO} 3-2$ transition.

To explain the size of the discrepancy between theoretical and observed $X$ values we consider the intensity variation within our simulated maps as a function of the column density. Figure 8 shows the ratio between the observed line integrated intensities and the optically thin LTE emissivity reflecting the column density for all points of a map in different transitions. 
In the ${ }^{13} \mathrm{CO} 1-0$ transition we find a broad range of column densities where the ratio is approximately constant at about 0.4 corresponding to an $X$ factor of $1.5 \times 10^{21} \mathrm{~cm}^{-2}\left(\mathrm{~K} \mathrm{~km} \mathrm{~s}^{-1}\right)^{-1}$ at the ${ }^{13} \mathrm{CO}$ molecular abundance of $1.5 \times 10^{-6}$. At column densities above $3 \times 10^{22} \mathrm{~cm}^{-2}$ the lines are saturated due to optical depth effects. The intensity remains approximately constant corresponding to an almost linear decay in Fig. 8 and a linear increase of the $X$ factor. At abundances typical for ${ }^{12} \mathrm{CO}$ optical depth effects dominate already at small column densities providing a monotonous decrease of the intensity ratio with column density. However, complete saturation of the emission, characterised by a linear behaviour, also occurs only at column densities above $10^{22} \mathrm{~cm}^{-2}$. Translating the plot into $X$ factors with a ${ }^{12} \mathrm{CO}$ abundance of $10^{-4}$ we obtain $0.47 \times 10^{20} \mathrm{~cm}^{-2}\left(\mathrm{~K} \mathrm{~km} \mathrm{~s}^{-1}\right)^{-1}$ at the lowest densities, $4.7 \times 10^{20} \mathrm{~cm}^{-2}\left(\mathrm{~K} \mathrm{~km} \mathrm{~s}^{-1}\right)^{-1}$ at a column density of $10^{22} \mathrm{~cm}^{-2}$, and much higher values in the saturated dense regions. The situation differs for higher transitions. In the ${ }^{13} \mathrm{CO} 3-2$ map we find an almost linear increase of the line intensity relative to the LTE value for low column densities because the line is subthermally excited throughout most parts of the cloud. For large column densities saturation sets in so that there is only a small column density range with a constant $X$ factor. Thus it is not possible to provide a unique $X$ factor to derive column densities from line intensities valid over different parts of a molecular clouds.

This is in contrast to several observationally based investigations showing that for certain regions the molecular line brightness reflects quite well the density structure measured by dust continuum, extinction maps, X-ray or gamma-ray emission. The contradiction may be partially due to insufficient resolution. In Fig. 9 we show the line intensity integrated over the whole map relative to the LTE brightness when scaling the density cube with different factors to obtain different average densities. The initial and the collapsed phase of the reference model behave completely different. If dense cores have formed after one free-fall time the integrated intensity is a very good tracer for the total mass in the cube. The specific line intensity varies by less than a factor three for all lines. In contrast, the high- $J$ lines in the model without dense cores are hardly excited at small density scaling factors leading to a steep increase of the specific intensity with increasing density. In the two lower transitions we can well distinguish the density range of subthermal excitation with increasing relative intensities from the regime of decreasing intensities, where optical depth effects dominate. Nevertheless there is no clear separation between both effects. Saturation by large optical depths takes over before the line excitation is in thermal equilibrium with the gas, so that the observable line intensity is always below the theoretical value for optically thin LTE emission.

We find the same behaviour for all other models. In case of a log-normal distribution of densities, which occurs in the turbulence simulations before gravitational collapse, the integral intensity in the lines relative to the LTE emissivity increases at low column densities and decreases for high column densities. The peak density depends on the transition and the width of this function depends on the width of the internal density distribution. A broad density distribution can lead
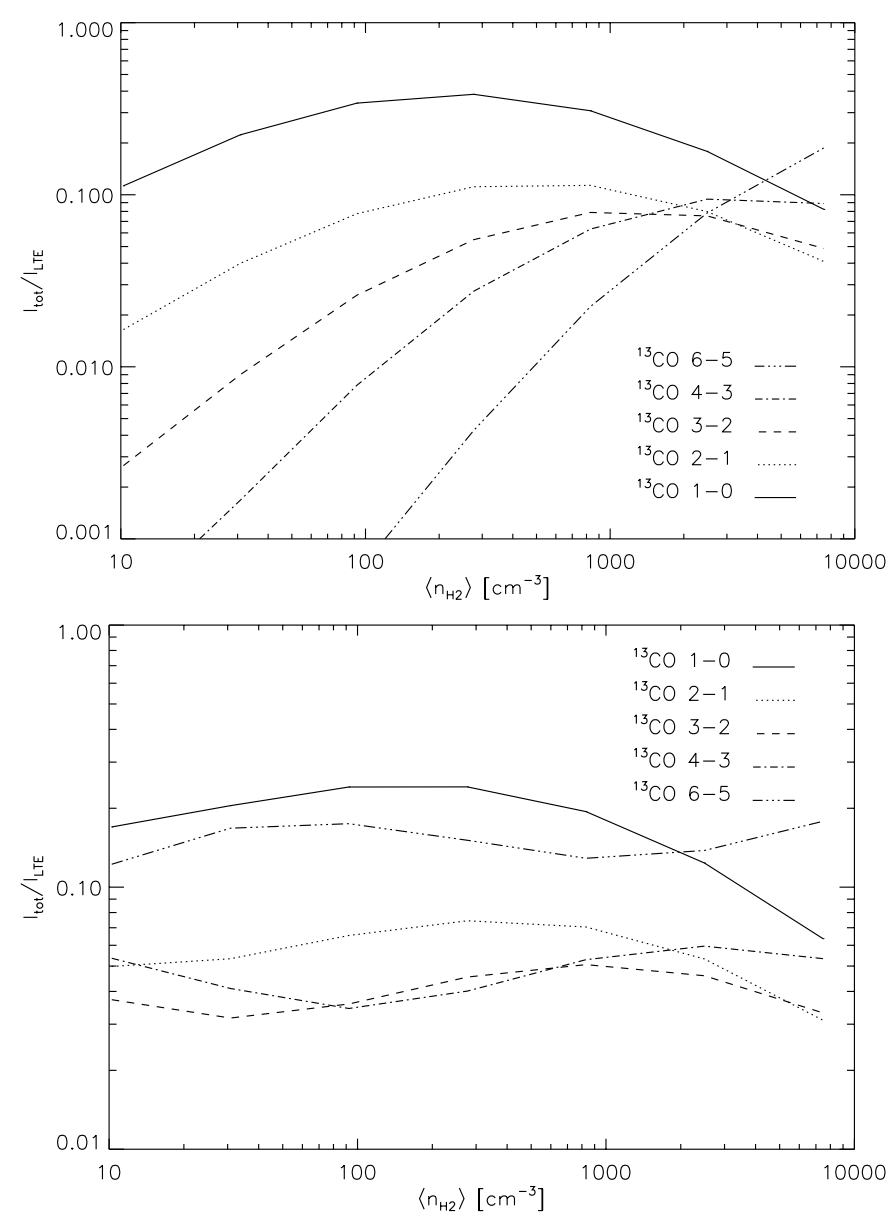

Fig. 9. Integrated line intensity of the total map relative to the intensity that the optically thin cloud with LTE excitation would provide as a function of the average density used to scale the cloud model. The upper plot is computed for the initial cloud model, the lower plot represents the cloud model with gravitationally collapsed cores.

to a broad peak providing an almost constant ratio over a large range of densities. In all hydrodynamic or magnetohydrodynamic simulations considered here the peak is relatively narrow, so that the ratio between the integrated line intensity and the LTE emissivity is quite sensitive to the actual density scaling. In case of a high density tail in the distributions which is produced by gravitational collapse the few dense clumps control the radiation field although they may contain only $10 \%$ of the total gas mass. The influence of non-LTE excitation at low densities and optical depth effects at high densities cancel each out so that the line intensity is proportional to the total mass in the clouds within a broad density range.

Thus two ways may have mislead observers to the conclusion that the $X$ factor is constant within molecular clouds. Each line has a density range where its intensity per column density is relatively constant. This range may be relatively broad like in the above example for the ${ }^{13} \mathrm{CO} 1-0$ intensities at low densities or quite narrow as in the corresponding ${ }^{13} \mathrm{CO} 3-2$ case. On the other hand observations with a finite telescope beam averaging over an area containing a wide distribution of column densities can also mimic a constant $X$ factor. In neither case we can use the $X$ factor derived for a certain region of a cloud to 

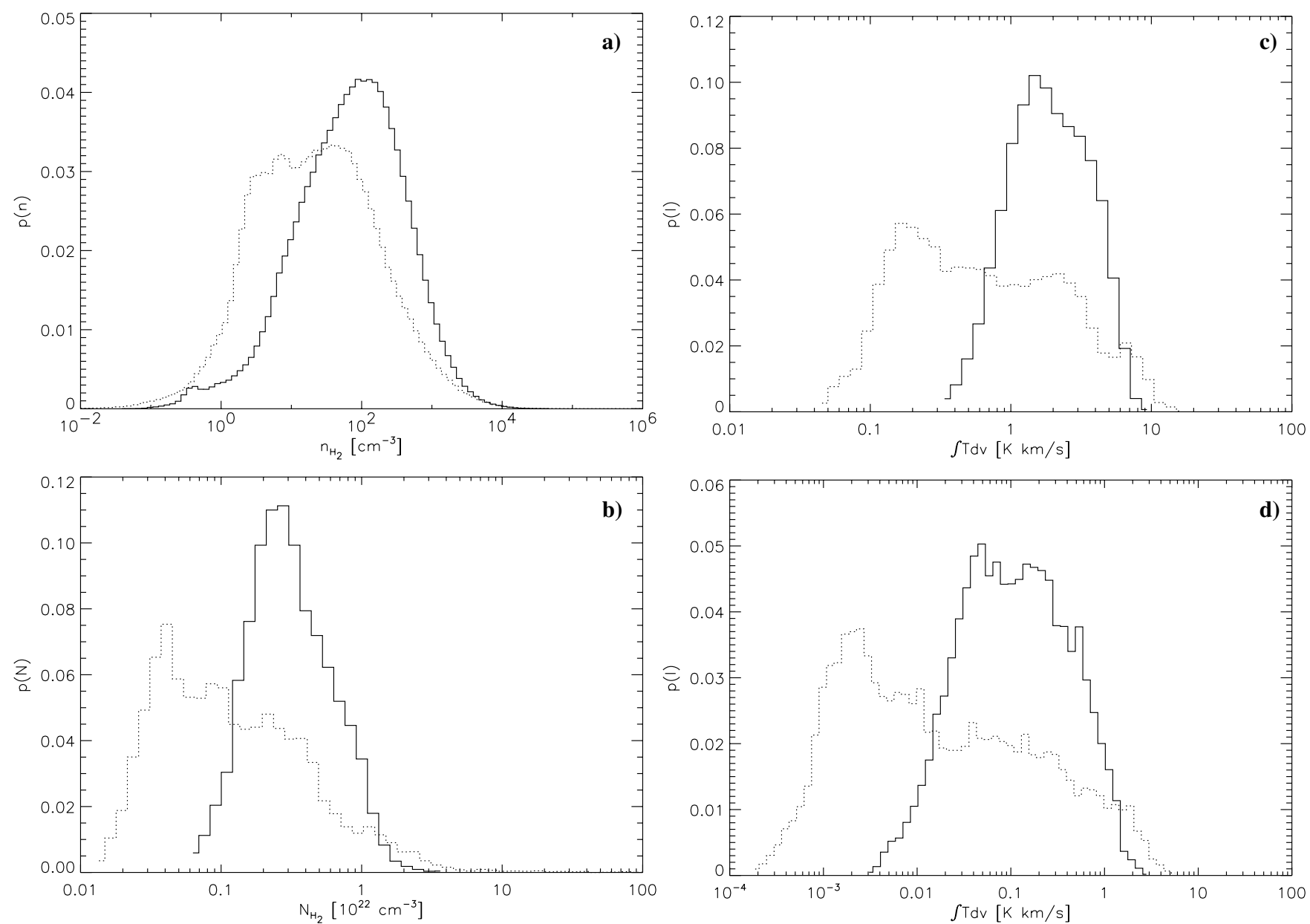

Fig. 10. Probability distribution functions of the density cubes a), of the corresponding column density maps b), and of the integrated line intensities in ${ }^{13} \mathrm{CO} 1-0$ c) and 3-2 d). The solid line represents the turbulence model before the onset of gravitational collapse, the dotted line is after about one free-fall time.

derive masses or column densities outside of this region. With this conclusion the justification of the $X$ factors applied for certain molecular clouds should be reconsidered for every single observational set.

\subsection{Intensity distributions}

In a next step we consider the intensity distributions in the maps to study their relation to the density distributions in the data cubes. Padoan et al. (1997) compared distributions of the density in turbulence simulations with distributions of the column density obtained by extinction measurements and Falgarone et al. (1998) discussed the corresponding distributions of CO line intensities. A comparison of the distributions in different models is given by Klessen et al. (in prep.).

In Fig. 10 we show the histograms for distributions in the reference model at the beginning of gravitational collapse and after one free-fall time, when $30 \%$ of the mass has turned into collapsed cores. On the left hand side, we indicate how the simple projection of the density structure into column densities changes the distributions. The dynamical range covered by the column density distributions is reduced by one order of magnitude relative to the density distributions.
The close-to-Gaussian shape of the initial density distribution is turned by the projection into a highly asymmetric distribution with a steep decay at small and a shallow tail at high column densities. The gravitational collapse produces few regions with very high density which are hardly visible in the density distribution but pronounced as a high-density tail in the column density distribution. As the collapse lowers the average density for most of the model volume we find a shift and broadening clearly visible in all distributions. On the right hand side of Fig. 10 we plot the corresponding distributions of the line integrated intensity measured either in ${ }^{13} \mathrm{CO} 1-0$ or in ${ }^{13} \mathrm{CO} 3-2$. In both distributions the high density tails are truncated due to optical depth effects, resulting in steep wings on both sides of the distributions. The subthermal excitation in 3-2 however stretches the intensity distribution to cover a much broader range than the column density distribution.

For a quantitative comparison we have summarised the standard deviations of the logarithmic distribution functions of each map in Table 2. In general, optical depth effects compress the intensity distribution by suppressing the high-density wing, reducing the contrast in dense parts of the cloud. At ${ }^{12} \mathrm{CO}$ abundances this effect dominates the whole intensity distribution. The low density wing of the intensity distribution is stretched 
Table 2. Logarithmic standard deviations of the density and intensity distributions in the different maps of the reference turbulence model.

\begin{tabular}{lcccccc}
\hline \hline time step & density cube & column density & ${ }^{13} \mathrm{CO} 1-0$ & ${ }^{13} \mathrm{CO} 3-2$ & ${ }^{12} \mathrm{CO} 1-0$ & ${ }^{12} \mathrm{CO} 3-2$ \\
\hline$t=0$ & 0.77 & 0.29 & 0.28 & 0.57 & 0.15 & 0.21 \\
$t \approx \tau_{\text {ff }}$ & 0.88 & 0.53 & 0.57 & 1.01 & 0.24 & 0.42 \\
\hline
\end{tabular}

relative to the column density distribution by subthermal excitation, enhancing the contrast in the map. Altogether, it is hardly possible to deduce the original density distribution from intensity histograms. Moreover, the intensity histograms of observed data are limited by noise. It is impossible to derive the shape of the distribution below the noise level. Thus one needs high signal-to-noise observations in order to measure the actual width of the intensity distribution so that on can distinguish between a translucent turbulent medium and a collapsed medium.

\subsection{Intensity scaling relations}

The $\Delta$-variance introduced by Stutzki et al. (1998) is a general method to measure the relative structural variation on different spatial scales within any data set. Bensch et al. (2001) have shown that it can be used to characterise the scaling relations in observed molecular cloud maps and Mac Low \& Ossenkopf (2000) applied it in an equivalent way to hydrodynamic and magneto-hydrodynamic turbulence simulations allowing a direct comparison with observations.

For observed or simulated molecular cloud maps the $\Delta$-variance is computed by convolving the map with a normalised, radially symmetric "French hat" wavelet of varying size and measuring the variance of the convolved map. For a comprehensive overview on the mathematical details we refer to Stutzki et al. (1998) and Zielinsky \& Stutzki (1999). The resulting $\Delta$-variance as a function of the filter size $\Delta s$ measures the amount of structural variation on that scale. It provides a clear spatial separation of various effects influencing observed structures such as noise or a finite observational resolution. If the map is characterised by a power-law power spectrum $P(|k|) \propto|k|^{-\beta}$, the $\Delta$-variance also follows a power spectrum $\sigma_{\Delta}^{2} \propto \Delta s^{\alpha}$ with $\alpha=\beta-2$.

Bensch et al. (2001) computed $\Delta$-variances for several molecular cloud maps observed in low- $J$ transitions of CO isotopes. They are close to power laws were the exponent $\alpha$ depends only on the size range considered and not on the starforming activity in some of the clouds. On scales above $1 \mathrm{pc}$ Bensch et al. obtained $\alpha \approx 0.6$, on scales below 1 pc $\alpha \approx 1.0$, and below 0.1 pc $\alpha \approx 1.3$.

Mac Low \& Ossenkopf (2000) used the $\Delta$-variance to compare the density scaling in molecular cloud simulations with the intensity scaling of molecular line observations assuming that the integrated intensity is a good tracer for the column density. This has to be repeated now taking radiative transfer effects into account.

In Fig. 11 we show the $\Delta$-variance plots of the molecular line maps computed for the reference model at the onset of gravitational collapse and after one free-fall time. We analyse the maps of different ${ }^{13} \mathrm{CO}$ transitions partly plotted in Fig. 7.
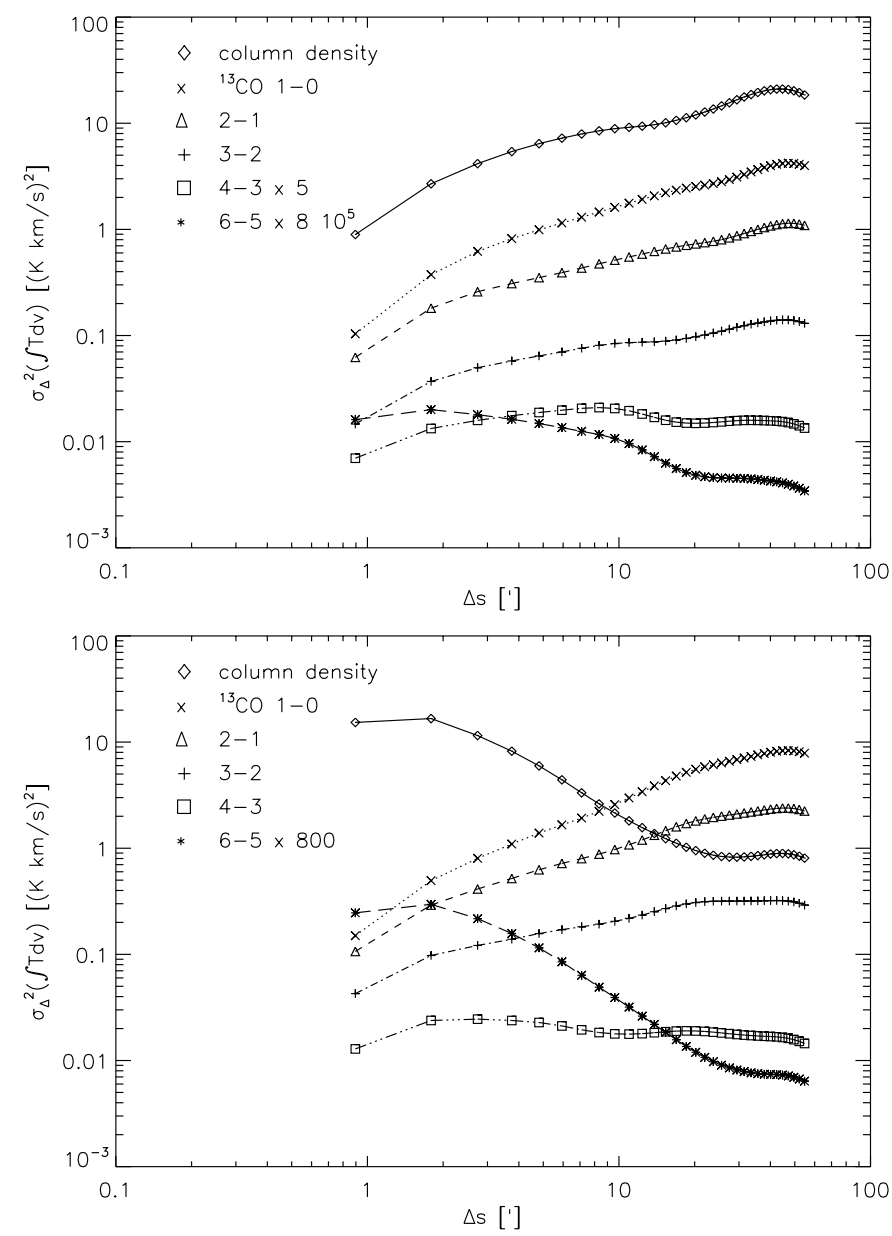

Fig. 11. $\Delta$-variances of the integrated maps obtained from the turbulence model cubes in the different transitions of ${ }^{13} \mathrm{CO}$. The upper plot represents the model before gravitational collapse, the lower plot is computed after about one free-fall time. For comparison the corresponding graphs for the column density maps are added in arbitrary units. The graphs for the highest transitions are shifted by the indicated values for a better visibility of the relative scaling behaviour.

On top of each plot the $\Delta$-variance of the corresponding column density map is added for a better comparison.

In the initial state, the $\Delta$-variance of the density structure can be approximated by a power law with exponent $\alpha=0.5$ in the size range between the numerical dissipation scale at about $3^{\prime}$ and about one third of the total cube size, i.e. $40^{\prime}$. The $\Delta$-variance is no perfect power law, but the deviations from a power law would be hardly detectable in observational data (Bensch et al. 2001). This $\Delta$-variance behaviour indicates a data set that is dominated by a large scale structure (limited here by the periodic boundary conditions of the simulations) and has a self-similar scaling down to the dissipation limit. The maps in the lower three ${ }^{13} \mathrm{CO}$ transitions reflect the scaling of 

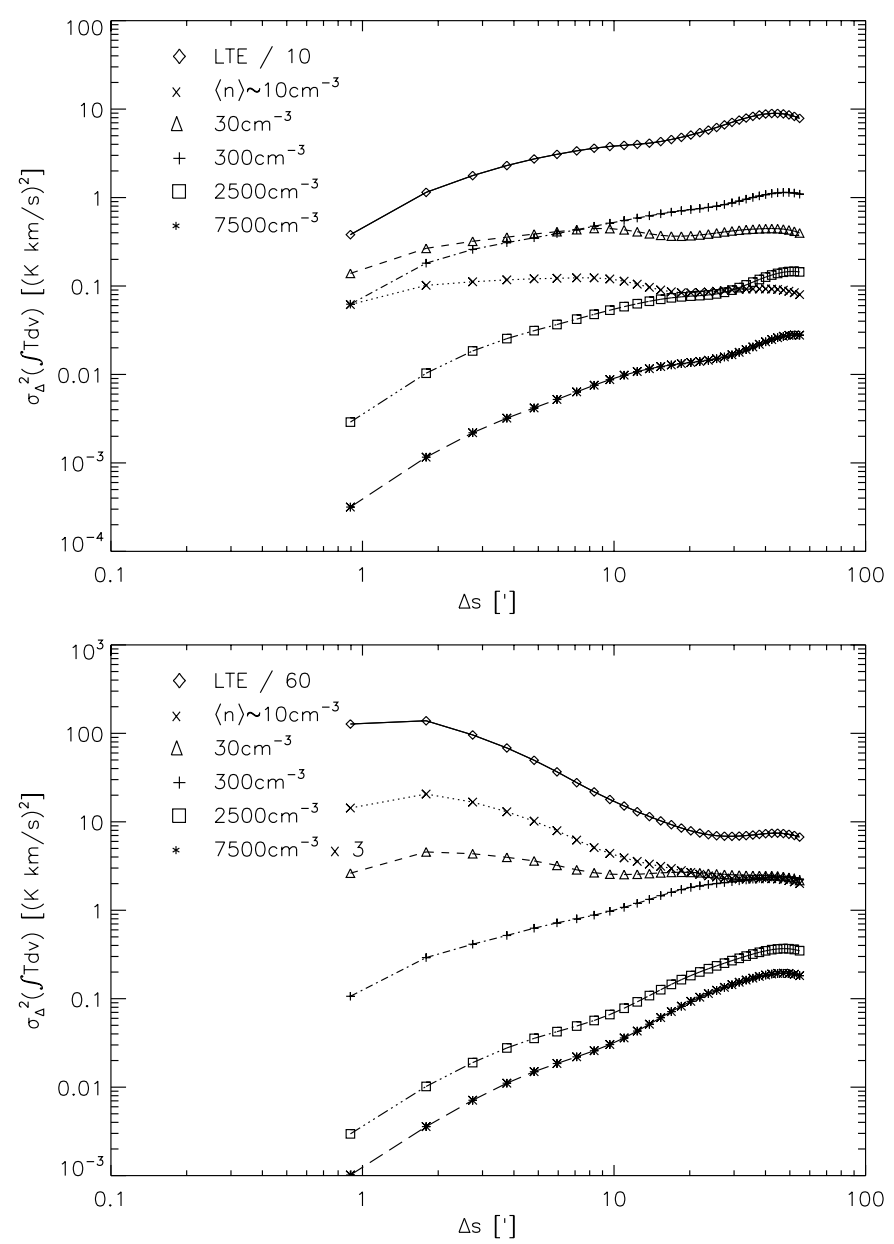

Fig. 12. $\Delta$-variances of the integrated maps in ${ }^{13} \mathrm{CO} 2-1$ obtained from the turbulence models applying different factors for the density scaling. The models are the same as in Fig. 11.

the density structure quite well, but with a slope that is steeper by 0.1 in the $1-0$ transition and shallower by 0.1 in the $3-2$ transition. The higher transitions, however, do no longer reflect the underlying densities. The 4-3 map shows about the same amount of structure on each scale and the 6-5 map is completely dominated by small scale structure given by the few dense regions in the model. In the collapsed state the structure in the low- $J$ maps is very similar although the column density map has completely changed. These transitions trace the lowdensity gas which is hardly influenced by the formation of the dense cores. The general trend of a slightly decreasing slope when going from the $1-0$ to the $3-2$ transition is also preserved but the change is somewhat larger. The exponent varies between 0.8 and 0.4 here. Only the 6-5 transition reflects the true density structure which is dominated by the few dense cores in this case.

To test whether this behaviour is specific to the conditions considered we have changed the scaling factor for the gas density. In Fig. 12 we show the $\Delta$-variance plots for the ${ }^{13} \mathrm{CO} 2-$ 1 maps in the two time steps from Fig. 11, when scaling the gas density with different factors. The standard scaling provides an average gas density of $275 \mathrm{~cm}^{-3}$. All intensity maps are normalised to this density before the $\Delta$-variance analysis.
To represent the column density maps we add the graphs for the ${ }^{13} \mathrm{CO}$ map assuming optically thin LTE projection.

The change of the slopes of the $\Delta$-variances with decreasing average density is similar to the change in the maps taken with increasing transition number $J$. As the critical density of the different transitions grows with $J$ increasing the density or decreasing $J$ have the same effect. When the density of the data cube is enhanced by a factor 30 the $\Delta$-variance slope of the ${ }^{13} \mathrm{CO}$ maps is 0.7 before gravitational collapse and 1.1 after the onset of collapse, i.e. somewhat steeper than in the 10 transitions when computed for the normal density. Down to an average density of $300 \mathrm{~cm}^{-3}$ we get only small changes in the slope of the $\Delta$-variance similar to the changes between the three low- $J$ transitions. Below $100 \mathrm{~cm}^{-3}$ the ${ }^{13} \mathrm{CO} 2-1$ transition is, however, hardly excited in the main part of the cloud, so that only the densest parts produce a noticeable emission leading to a constant or decaying $\Delta$-variance. The true density structure has only little effect on this behaviour.

Equivalent plots for a variation of the molecular abundances (not shown) are quite similar to the plots for varying density. This is reasonable because an increasing abundance leads to radiative trapping and hence to the excitation of a larger volume of the cloud model. At ${ }^{12} \mathrm{CO}$ abundances the $\Delta$-variance slopes are always somewhat steeper than computed for ${ }^{13} \mathrm{CO} .{ }^{12} \mathrm{CO}$ maps corresponding to Fig. 11 show large scale structure in the four lower transitions with slopes between 0.9 and 0.5 . Only the $6-5$ transition is dominated by small-scale structure there. When the abundance is reduced by a factor $30-100$ relative to the standard ${ }^{13} \mathrm{CO}$ value the $\Delta$ variance of the maps in the low- $J$ transitions turns flat independent of the time step in the model.

Hence, the $\Delta$-variance of the maps taken in low- $J$ transitions always measures the structure of the thin extended gas, not allowing a direct conclusion on the actual density structure. We cannot expect to retrieve the true density structure from any molecular line map. The change of the $\Delta$-variance slope between maps taken in different low- $J$ transitions, however, gives a hint on the distribution of densities. In all models without dense cores the variation in the slopes between the 1-0 and the $3-2$ transitions of ${ }^{13} \mathrm{CO}$ falls below 0.3 whereas the change exceeds 0.4 if a considerable fraction of dense cores contributes to the excitation pattern. These numbers hold as well for ${ }^{12} \mathrm{CO}$ if we compare maps in the 1-0 and the 4-3 transitions.

It is however, difficult to measure the slope of the $\Delta$-variance in observational data with an uncertainty below 0.1 . In Fig. 13 we compare the $\Delta$-variance plot of the ${ }^{13} \mathrm{CO} 2-1$ map already shown in Fig. 11 with the $\Delta$-variance after convolving the map with a Gaussian beam of twice the cell size and after adding white noise with a signal-to-noise ratio of 20 , which is quite typical for molecular line observations. Although both observational effects act only at small lags, one is easily forced to see a somewhat steeper $\Delta$-variance spectrum in the noisy data corresponding to real observations. Bensch et al. (2001) have shown that it is in principle possible to correct the $\Delta$ variance both for the beam smearing and noise if one assumes that the spectrum follows a power law. Regarding the error bars of this correction we come to the same conclusion as for the intensity distributions. We need very high signal-to-noise 


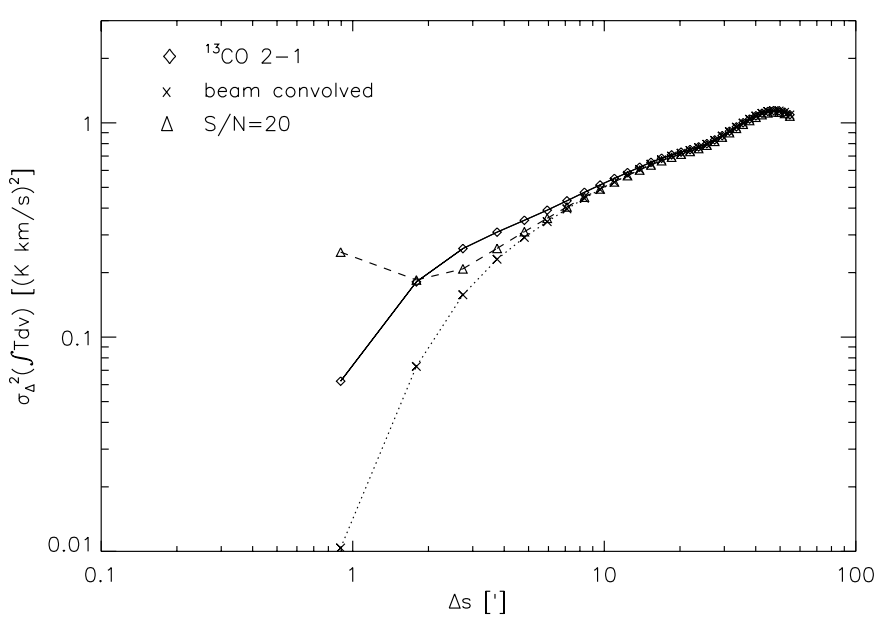

Fig. 13. $\Delta$-variance measured in the ${ }^{13} \mathrm{CO} 2-1$ map of the reference model at $t=0$ when we consider the intensities on the original grid, convolve the map with a beam of twice the cell size, and when we apply additional noise with a $S / N$ of 20 .

observations to get sufficiently reliable exponents to deduce the existence of dense cores from low- $J$ observations in $\mathrm{CO}$ isotopes.

\section{The velocity structure}

\subsection{Line profiles}

The radiative transfer will also affect the signatures of the velocity structure observable in molecular lines. A first impression comes from the inspection of the shape of the line profiles and their spatial variation across the maps. Figure 14 shows the line profiles in the reference model on three points along a central cut together with the profiles at the positions of the maximum absolute and integrated line temperature and the average line profile. Here, beam convolution is taken into account, leading to a small blurring of the profiles, but no noise is added. Real observations with a finite signal-to-noise ratio are not able to recover all the details in the profiles shown here so that we will not discuss small ripples in the profiles but concentrate on the general structure.

At most points of the map we find irregular line shapes consisting of two or three components which can be distinguished by eye. The roughness of the line profiles can be reduced relative to Fig. 14 by assuming observations with larger telescope beams but a larger convolving beam does not change the general broken structure of the line profiles. CO observations show comparable line profiles in regions with heavy and crowded star-formation, whereas they typically reveal smooth profiles without substructure for more quiescent regions (e.g. Kutner et al. 1997; Goodman et al. 1998). This holds as well for the Polaris Flare observations mentioned above where the line profiles are close to Gaussian on most points of the maps with some additional wing contribution and weak signs of selfabsorption. We find such smooth profiles only in turbulence models driven on small scales, consisting of many weak shock structures along each line of sight. However, the small-scale driven turbulence models are inconsistent with the observed

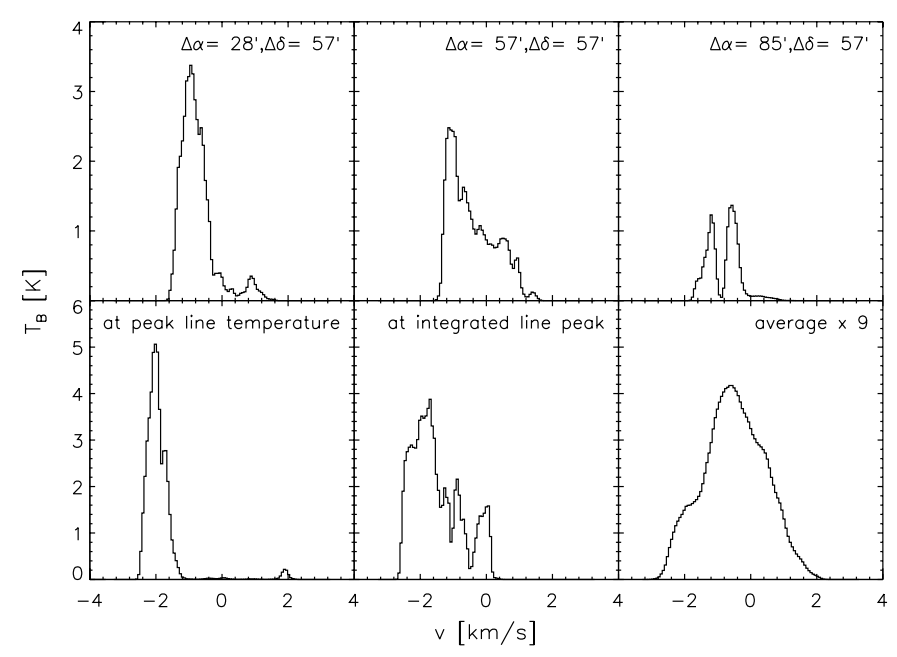

Fig. 14. Line profiles observed in ${ }^{13} \mathrm{CO} 2-1$ at three positions of a horizontal cut through the map shown in Fig. 5 and at the positions of the peaks in the line temperature and the integrated line temperature together with the average line profile.

intensity scaling behaviour which is only reproduced by turbulence models driven on large scales. All large-scale driven models show broken, irregular line profiles on a majority of positions within the maps. The formation of few large intensity structures corresponding to the major parts of observed molecular clouds is always accompanied by the formation of few major velocity modes which are well separated in the line profiles leading to their broken, irregular shape.

A possible solution to this puzzle is turbulent substructure on scales below the resolution of a single pixel considered in the simulations. With additional sub-pixel turbulence the effective velocity dispersion within each pixel is much larger than thermal. Although this violates the self-consistency of the turbulence models, we have tested this case by adding a turbulent velocity dispersion within each pixel which was either constant at up to seven times the thermal dispersion or depending on the local density $\Delta v_{\text {local }} \propto n^{-1 / 2}$. The resulting line profiles are much smoother than in Fig. 14 but the general appearance of the broken line profiles consisting of several components is not changed. Gaussian line profiles occur only if we add local velocity dispersions close to the total velocity dispersion in the turbulence model. Padoan et al. (1998) proposed to add local velocity dispersions according to the velocity gradients between neighbouring pixels. Figure 1 demonstrates, however, that for most points the gradients between neighbouring pixels are not large compared to the thermal line width, so that this does not help. We are left to conclude that we do not yet have a self-consistent turbulence model explaining the observed line profiles in quiescent regions.

Nevertheless, we will continue to discuss the influence of radiative transfer effects on the measurable velocity structure as the variety of models allows to draw some general conclusions. In Fig. 15 we show the ${ }^{13} \mathrm{CO} 2-1$ line profiles for the large-scale driven model after one free-fall time of gravitational collapse. The comparison with Fig. 14 allows to identify the effect of dense cores on the line profiles. In Sect. 4 we demonstrated that dense cores are mainly visible in intensity maps of higher 


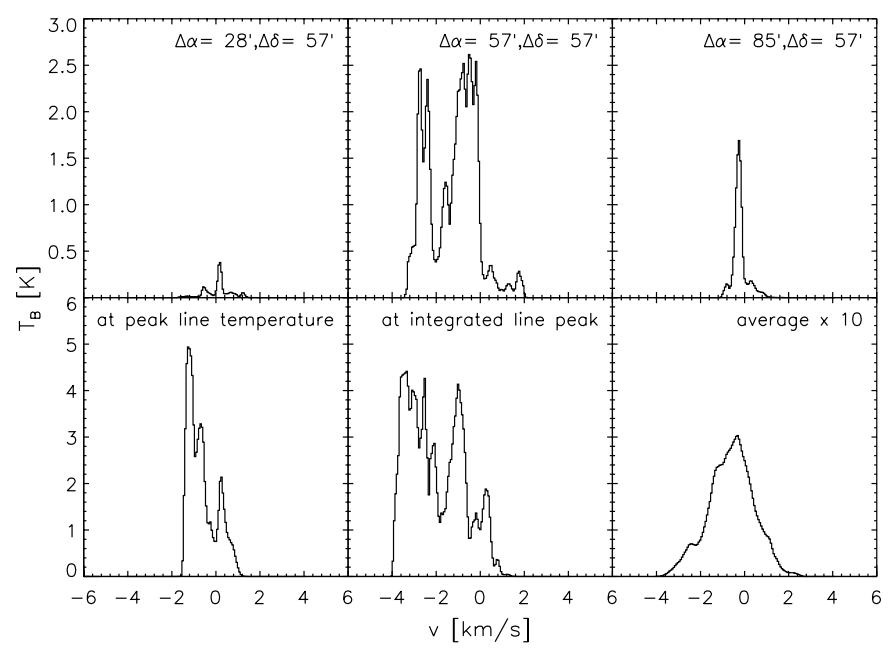

Fig. 15. ${ }^{13} \mathrm{CO} 2-1$ line profiles for the turbulence model after the formation of cores by gravitational collapse.

transitions. Here, we see that they also leave signatures in the line profiles of the 2-1 transition. As the cores are coherent in velocity space they show up as narrow spikes in the profiles. Although it is impossible to conclude the existence of dense cores from any single line profile, the general structure of the line profiles across the map provides a clear indication of dense cores if they break up into many narrow spikes. This should be measured by parameters for the smoothness of line profiles as introduced by Tauber (1996). Then the existence of cores may be inferred even from low- $J$ observations if the molecular lines have moderate optical depths outside of the cores. In our models the cores are undetectable in low- $J$ profiles of ${ }^{12} \mathrm{CO}$ but clearly visible in the ${ }^{13} \mathrm{CO}$ lines as demonstrated above.

\subsection{The average line profile}

In contrast to the line profiles at single positions in the maps Figs. 14 and 15 show that the average profiles are smooth with wings which are close to Gaussian. The central parts may be described as somewhat distorted Gaussians.

There is a long lasting discussion whether the average profile of molecular lines may be used as an estimate for the probability distribution function (PDF) of velocities in molecular clouds (see e.g. Falgarone et al. 1998; Miesch \& Bally 1994; Ossenkopf \& Mac Low 2002). Here, we test for which lines this may hold and how radiative transfer effects change the average line profile.

In Fig. 16 we show the map averaged profiles of ${ }^{13} \mathrm{CO} 4-3$ for the small scale driven turbulence model S02 before gravitational collapse. We select this model here in contrast to the reference model because the small scale-driving produces many small turbulent eddies providing a good statistical sample so that the global velocity PDF is close to Gaussian. In the large scale driven models, the PDFs are distorted by the statistical variation of the few largest modes (Ossenkopf \& Mac Low 2002) which is also visible in the average line profiles in Figs. 14 and 15 In the irregular PDFs the changes discussed here may be somewhat hidden in the statistical

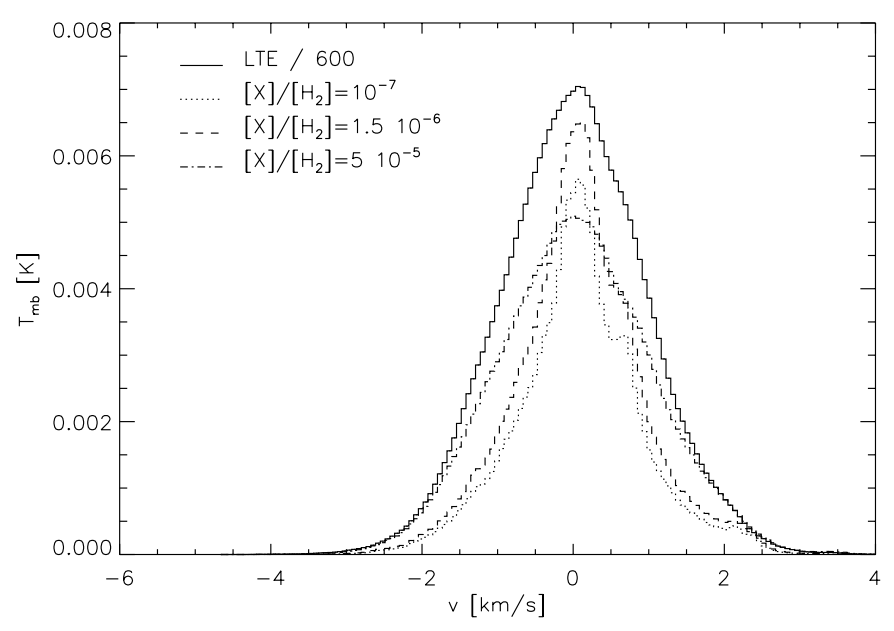

Fig. 16. Average line profiles in ${ }^{13} \mathrm{CO} 4-3$ for the small scale-driven model S02 when varying the assumed molecular abundance. The line temperatures are normalised to the standard abundance of $1.5 \times 10^{-6}$. The profile for the LTE projection is rescaled by a factor $1 / 600$.

variation. Thus, we chose the Gaussian PDF case for educational purposes but want to stress that the conclusions hold also for the other models. We show the 4-3 line, because it undergoes a transition from a regime of negligible collisional excitation to a regime dominated by optical depth effects when we change the assumed molecular abundance of ${ }^{13} \mathrm{CO}$ around the standard value. The solid line in the figure represents the LTE projection, i.e. the actual density weighted PDF of the line-ofsight velocities.

We find two scenarios. At the two lower abundances, where the transition is subthermally excited and optically thin, only the central part of the profile is prominent as a narrow peak with broad, almost exponential, wings. At abundances close to the typical values for the main $\mathrm{CO}$ isotope the centre of the profile is suppressed by optical depth effects so that only the wings trace the true velocity PDF and the average profile is subGaussian. This behaviour hardly depends on the molecular cloud models used. Subthermal excitation tends to mimic PDFs with strong wings which might be misinterpreted as indicator for intermittency (Falgarone \& Phillips 1990) or vorticity (Ballesteros-Paredes et al. 1999a). Optical depth effects always broaden the profiles so that the line wings appear steeper than in the original PDF when considered relative to the centre of the distribution. This has to be taken into account for all low- $J$ transitions in ${ }^{12} \mathrm{CO}$ and also for many observations in ${ }^{13} \mathrm{CO}$. Thus the detection of sub-Gaussian average line profiles in the ${ }^{12} \mathrm{CO}$ observations of the Polaris Flare discussed by Ossenkopf \& Mac Low (2002) may well be attributed to line saturation in ${ }^{12} \mathrm{CO}$ instead of a sub-Gaussian velocity PDF of the molecular cloud.

Beside the wing behaviour different transitions also show a different sensitivity to the formation of dense cores. In the models with collapsed cores the average line profile in the 6-5 transitions consists of sharp peaks produced by the cores and a broad weak wing contribution. The true density weighted velocity PDF, however, is rather similar to the turbulent models without collapse because the cores are formed as parts of 
larger shock structures with a broad internal velocity gradient. In the LTE projection the cores are only visible as small peaks on top of a broad continuous profile. In the average profile of low- $J$ transitions they are almost completely hidden as shown in Fig. 15.

The best tracer for the velocity PDF is a rare isotope with low critical density. The $\mathrm{C}^{18} \mathrm{O} 2-1$ average line is a good measure for the PDF in all our simulations. As the lines from rare isotopes are often quite weak, preventing an efficient observation, one can also combine results from different isotopes or different transitions to scan different parts of the profile as proposed already by Falgarone et al. (1998). In a consistent observational set it should be feasible to combine the data of the three main $\mathrm{CO}$ isotopes to trace the different parts of the line profile using ${ }^{12} \mathrm{CO}$ only for the outer wings, $\mathrm{C}^{18} \mathrm{O}$ for the line centre, and ${ }^{13} \mathrm{CO}$ for the transitional velocities.

\subsection{Velocity scaling}

The $\Delta$-variance used to measure the intensity scaling in Sect. 4.4 may be used as well to measure the spatial scaling behaviour of the velocity structure if we consider maps of line centroid velocities. Ossenkopf \& Mac Low (2002) showed that the $\Delta$-variance is more sensitive to local changes in the velocity scaling behaviour than the size-linewidth relation or the structure function in centroid maps but has drawbacks when applied to noisy data. As we may omit noise in the model data we can use the square root of the $\Delta$-variance here to analyse the average velocity dispersion as function of spatial lag but the other methods might be preferable for a corresponding analysis of observed data.

The determination of the velocity scaling behaviour in the Polaris Flare observations and in turbulence models by Ossenkopf \& Mac Low (2002) showed that both types of data sets are consistent with a shock dominated medium where the exponent of the power spectrum is close to four and the velocity variation as function of lag follows $\Delta v \propto \Delta s^{\gamma}$ with $\gamma \approx 0.5$. This power law is broken above the driving length scale in the models and at scales of the overall molecular cloud size in the observations. Moreover the models showed a clear steepening of the $\Delta$-variance spectrum at the smallest scales due to the numerical dissipation.

To demonstrate how this result may be influenced by radiative transfer effects we show in Fig. 17 the $\Delta$-variance of the ${ }^{13} \mathrm{CO} 2-1$ line centroid maps for a large-scale driven hydrodynamic model before gravitational collapse. In contrast to the figures above, we have used here the grid-based model H01 because the velocity structure of the SPH models shows some artifacts resulting from a poor representation of the velocity dispersion in thin regions where few SPH particles are found (Ossenkopf et al. 2001b). The LTE projected velocity structure shows a power-law $\Delta v \propto \Delta s^{\gamma}$ with exponent $\gamma=0.47$ between the dissipation scale at about $3^{\prime}$ and an upper limit at about $30^{\prime}$ corresponding approximately to the shockdominated behaviour mentioned above. It is not clear why the upper limit appears at somewhat lower lags than in the intensity structure given e.g. in Fig. 12.

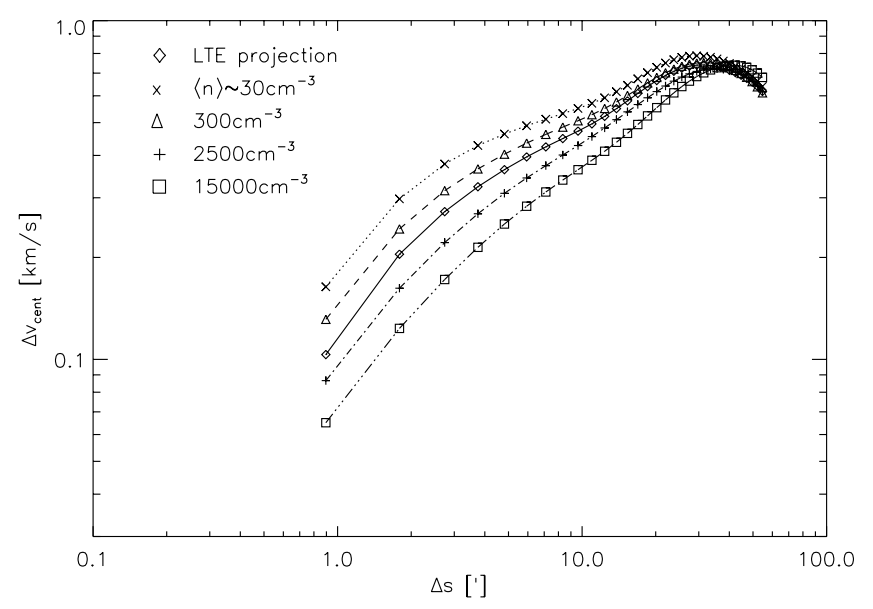

Fig. 17. Square root of the $\Delta$-variance of the line centroid velocities in ${ }^{13} \mathrm{CO} 2-1$ maps computed for the grid-based large-scale driven hydrodynamic model $\mathrm{H} 01$ when changing the applied density scaling factor.

Comparing the results from the ${ }^{13} \mathrm{CO}$ line maps with the projected LTE situation we find that at the average density of $30 \mathrm{~cm}^{-3}$, where the lines are subthermally excited, the slope of the curve is lowered to about 0.38 and the small curvature around $12^{\prime}$ is enhanced. At average densities above about $1000 \mathrm{~cm}^{-3}$, where optical depth effects are important, the slope is increased to about 0.55 and we obtain an almost perfect power law up to lags of about $40^{\prime}$. In models with even higher densities falling above $30000 \mathrm{~cm}^{-3}$ or when combining the highest density scaling in this plot with the abundance of ${ }^{12} \mathrm{CO}$ we get saturated flat-top line profiles in large parts of the map. Then all centroid velocity fluctuations on small scales are efficiently suppressed and the velocity $\Delta$-variance shows a sharp drop-off below about $10^{\prime}$. However, observations of tracers which are completely saturated throughout the map are not likely to be conducted so that the result is not shown here.

The figure corresponding to Fig. 17 after the onset of gravitational collapse shows a strong change of the velocity structure on large scales. Velocity fluctuations on distances above about $15^{\prime}$ are no longer traced by the line centroids as most of the mass is concentrated in shock regions and part already collapsed into cores. The $\Delta$-variance decays at larger lags. The structure on scales below about $8^{\prime}$ is hardly affected by the gravitational collapse. The radiative transfer effects are quantitatively equivalent to the situation in the initial turbulence model in Fig. 17.

When considering different transitions or different abundances the variation of the velocity centroid $\Delta$-variance follows the same scheme, but is in general less pronounced than for the density scaling variation. Insufficient collisional and radiative excitation at low densities, low abundances, or in high- $J$ transitions tends to flatten the spectrum. Saturation at high abundances or high densities results in a steepening of the $\Delta$-variance spectrum. At the normal density scaling the slope of the relation between size and velocity variation is only slightly modified. The Polaris Flare observations in different $\mathrm{CO}$ isotopes by Falgarone et al. (1998) do not show a clear change in the slope between the different isotopes. Regarding the typical 
error bars in the observational data (Ossenkopf \& Mac Low 2002) the small changes are hardly detectable in the total uncertainty of the slope as long as the molecular line maps are not dominated by saturated lines. Thus future observations should try to combine transitions which are partially saturated with transitions which are only excited in the dense regions. One has to keep in mind, however, that already moderate optical depth effects in low- $J$ transitions can effectively hide the dissipation limit in the velocity scaling spectrum as seen in Fig. 17.

\section{Differences between the models}

\subsection{The influence of turbulent driving}

All basic properties of the turbulence models used here are already discussed in the original papers introducing them (Mac Low et al. 1998; Mac Low 1999; Klessen et al. 2000; Heitsch et al. 2001). There is a large number of papers comparing the properties of different hydrodynamic and magnetohydrodynamic models (cf. e.g. Ostriker 1999). We will not perform the same comparison here, but we consider only differences between turbulence models which are especially prominent in the molecular lines maps.

Section 4 showed that the most prominent change of the molecular line maps results from the existence or absence of collapsed cores. Thus, different time steps of a collapsing model may produce a stronger change in the molecular line emission than the use of different models. Although the collapse heavily changes the overall excitation it is hardly traced in the lowest $\mathrm{CO}$ transitions. Here, the global turbulent driving is the dominant parameter. Different scales of the driving processes produce the same scaling behaviour in the low- $J$ molecular line maps that was discussed by Mac Low \& Ossenkopf (2000) for the density structure. The turbulence does not create any structure at scales above the driving wavelength. As the observations show approximately power-law $\Delta$-variance spectra for the molecular line maps increasing up to the size of the molecular clouds a good representation of these observations is only possible in models where most of the kinetic energy is injected on the scale of the overall cloud size.

The influence of a different strength of the turbulent driving was discussed by Mac Low (1999) and studied systematically by Padoan et al. (1997). They find that the standard deviation of the density distribution grows linearly with the rms Mach number of the turbulent flow. Comparing the models with variable driving Mach number H01, HC2, and HE2 we obtain about the same behaviour but a different prefactor depending on the details of the simulation. The simulations driven to give an rms Mach number $\mathcal{M}$ of 15 have substantially denser shock regions than the model shown in Sects. 4 and 5 which has $\mathcal{M}=10$.

In Fig. 18 we plot the $\Delta$-variance of ${ }^{13} \mathrm{CO}$ line intensity maps for the models HC2 and HE2 driven to obtain either an rms Mach number of 5 or of 15 . Their density distributions have logarithmic standard deviations of 0.47 and 1.13, respectively, i.e. below and above the value for the reference model given in Table 2. The figure includes the results for the 2-1 and the 4-3 transitions to demonstrate the behaviour of one line which starts to become optically thick and one

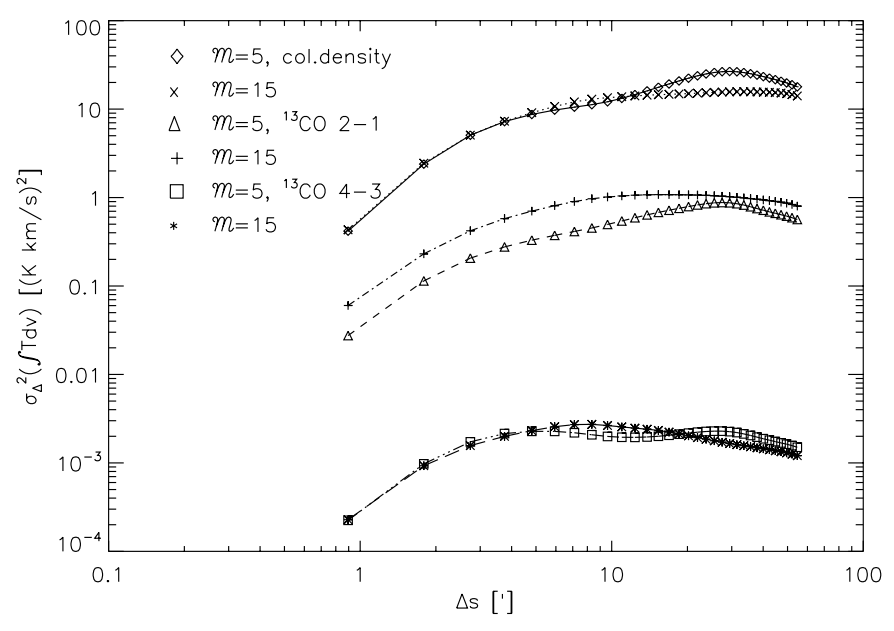

Fig. 18. $\Delta$-variance of the column density and the integrated ${ }^{13} \mathrm{CO}$ 2-1 and 4-3 maps for the large-scale driven hydrodynamic models $\mathrm{HC} 2$ and HE2 driven at different rms Mach numbers. The $\Delta$-variance for the column densities is scaled by an arbitrary factor.

line which is hardly excited in most parts of the cloud models. One top of the plots we have added the $\Delta$-variance spectra for the column density maps. The line-of-sight integration of the density structure in the column density maps hides the different widths of the density distributions. The absolute variation within the column density maps is about the same in both models. The stronger driving does not change the magnitude but the slope of the $\Delta$-variance. In the significant range of lags between $3^{\prime}$ and $40^{\prime}$ the average slope is reduced from about 0.5 in the $\mathcal{M}=5$ model to 0.35 at $\mathcal{M}=15$. The visual inspection of the column density maps shows a larger number of shock fronts in the model with the strong driving. The stronger driving does not produce denser shocks on all scales, but the interaction of the fast shocks disperses some large scale structures. Future investigations should clarify whether this effect is based on the limited dynamical range between the driving and the dissipation scale in the numerical simulation or whether a change of the driving Mach number results in a true change of the turbulent energy cascade.

The $\Delta$-variance plots for the molecular line maps provide a good reflection of the scaling behaviour of the column density structure but they show a slight decrease of the $\Delta$-variance slope with increasing transition number. The emission in higher transitions becomes less space filling but the long-range correlation of the structure is preserved as all dense spots are part of larger shocks also visible in the low- $J$ transitions. They behave different than the density enhancements due to gravitational collapse. Comparing Fig. 18 with Fig. 11 shows that gravitational collapse is always confined to small scales whereas stronger driving influences all scales.

As the influence of the driving Mach number on the density scaling is relatively small definite conclusions on the Mach number should come from the line profiles. In optically thin lines we recover the difference in the Mach number as the difference in the average width of the line profiles. In optically thick lines the difference is smaller, but the increase of the line width in the strong driven model is e.g. responsible for the 

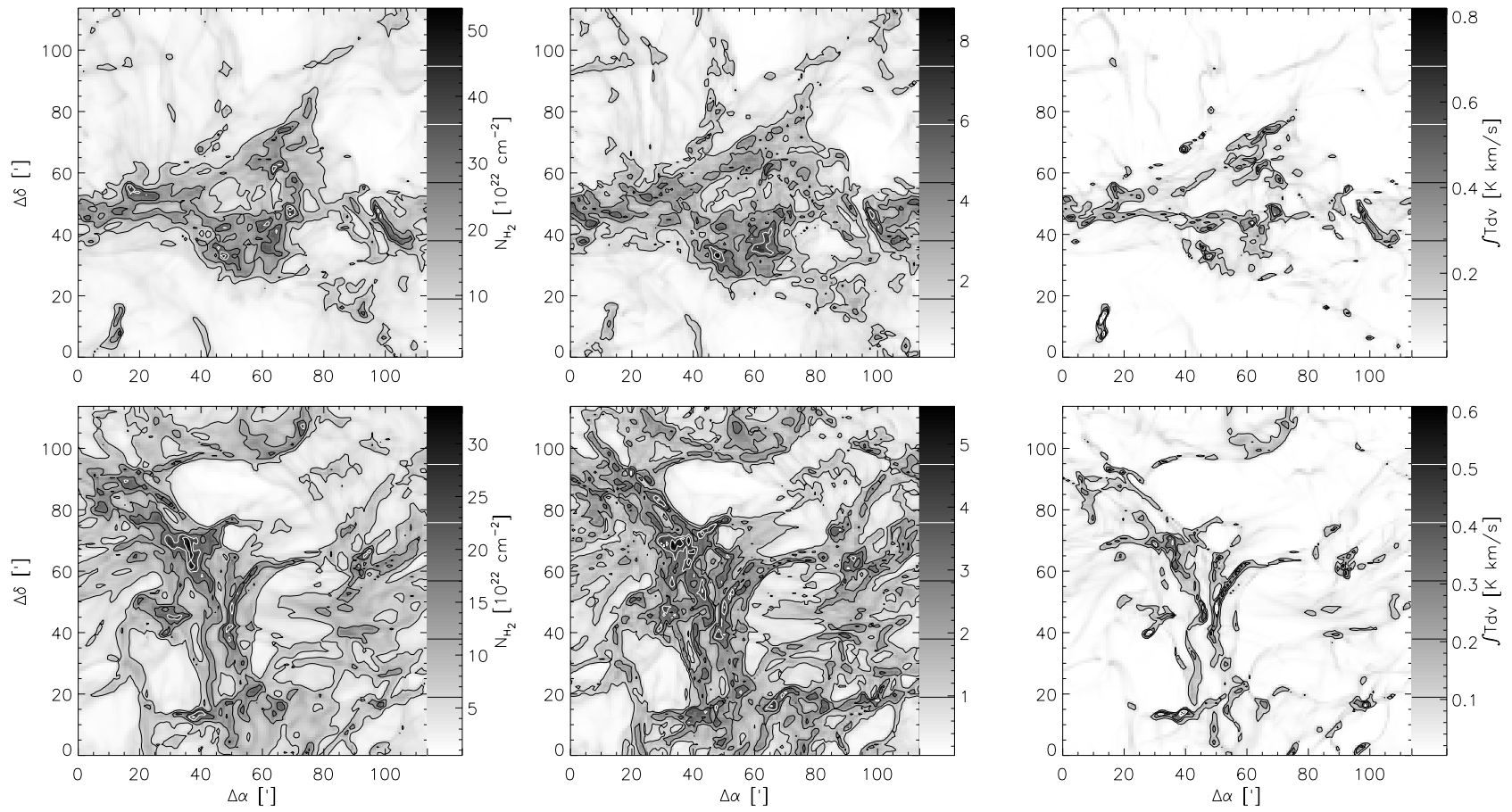

Fig. 19. Column density map (left panel) and ${ }^{13} \mathrm{CO} 2-1$ and 4-3 integrated line maps for the MHD turbulence model M01 perpendicular to the direction of the initial magnetic field (upper row) and parallel to this direction (lower row).

stronger variations of the integrated ${ }^{13} \mathrm{CO} 2-1$ line intensities visible in Fig. 18.

\subsection{Magnetic fields}

Another effect that is especially pronounced in molecular line maps is anisotropy introduced by the magnetic fields. Ossenkopf \& Mac Low (2002) found a clear anisotropy in the velocity structure of sub-Alfvénic turbulence. On contrary, in model M01, which has an rms turbulent velocity exceeding the Alfvén velocity, Heitsch et al. (2001) and Ossenkopf et al. (2001b) found that the magnetic field acts mainly as an additional pressure not introducing clear anisotropies in the velocity structure.

In Fig. 19 we demonstrate maps for model M01 when observing perpendicular and parallel to the direction of the original magnetic field. Already eye inspection shows clear differences between the two directions. When the line of sight is perpendicular to the field direction, the appearance of the maps is similar to the other large scale driven turbulence models. The somewhat more filamentary structure compared to the more clumpy structure in Fig. 7 is a general feature of the grid-based simulations (Klessen et al. 2000). The maps observed parallel to the initial magnetic field show a more extended structure which is composed of many warped filaments. The magnetic field tends to produce density structures with the shape of spirals. They penetrate each other less than the normal shocks filling almost the whole map area. This is also visible in the scaling behaviour. Figure 20 shows the $\Delta$-variance of the ${ }^{13} \mathrm{CO}$ line intensity maps and the column density maps in the two directions. We find a lack of large-scale structure in the line-of-sight

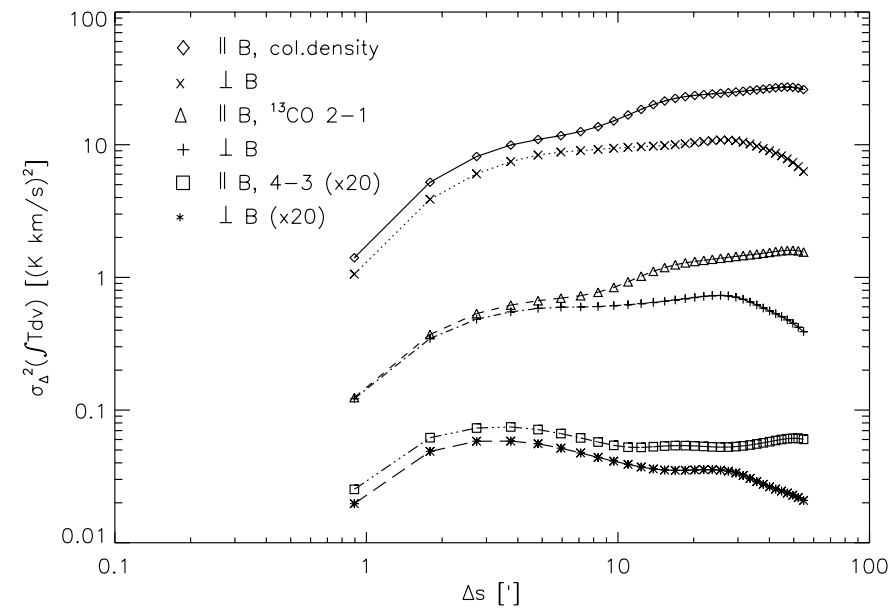

Fig. 20. $\Delta$-variance of the column density and the integrated ${ }^{13} \mathrm{CO} 2-1$ and 4-3 maps for model M01 with the line of sight perpendicular or parallel to the direction of the initial magnetic field.

direction parallel to the magnetic field that is also traced by all molecular lines. The average $\Delta$-variance slope in the significant range is reduced by 0.2 . At small lags both directions agree in their scaling behaviour. Thus the removal of large-scale structure by the magnetic field found in the three-dimensional density distribution by Mac Low \& Ossenkopf (2000) is confined to two directions because the magnetic field suppresses only perpendicular motions.

The anisotropy is hardly reflected in the line profiles. We find no significant differences in their shape between the two directions. The average line profiles show the same velocity dispersion. This explains why the anisotropy was not detected in the investigation of the velocity scaling behaviour 
by Ossenkopf et al. (2001b). Now, we have to state that even in case of low magnetic fields their direction introduces an anisotropy into the global structure which is partially retained during the turbulent evolution. From the observational constraint that molecular clouds show structures correlated on large scales with $\Delta$-variance slopes between 0.6 and 1.3 we can only exclude strong magnetic fields parallel to the line of sight, but cannot give any statement on the field in other directions. Assuming that we are not located in a preferred direction with respect to all clouds this means that either the fields are weak or substantially entangled within the clouds.

\section{Summary}

\subsection{The radiative transfer}

The computation of molecular line maps that would be observed from molecular cloud models needs a sophisticated radiative transfer model which takes the radiative interaction in the clouds into account. As the fully self-consistent integration scheme by Juvela (1997) is computationally very demanding and hardly suited to evaluate several hundred different cloud models we present and apply another code based on a twoscale approximation for the radiative interaction. The excitation by radiation from the vicinity of each point is treated by an adapted LVG approximation. Here, the strong density gradients in turbulent shocks are taken into account, but even the "ordinary" LVG approximation is still quite accurate although its underlying assumptions are no longer fulfilled. As the turbulent medium is isotropic on large scales and the radiative excitation integrates over all spatial directions and frequencies it is possible to treat the long-range radiative interaction by an average line profile introduced as external field into the local LVG approximation. Combining both approximations allows to compute the molecular line emission from cloud models containing $128^{3}-256^{3}$ pixels on a small computer within a few up to some ten minutes with an accuracy which is comparable to the calibration uncertainties of most molecular line observations.

\subsection{Properties of the molecular line emission}

With this radiative transfer model we evaluate a set of turbulence models to see how their properties are reflected in molecular line maps. The results provide rough guidelines for the interpretation of molecular line observations to deduce the true structure of the corresponding molecular clouds taking into account the general effect of radiative transfer.

Section 4 demonstrates that integrated molecular line intensities, independent of the particular transition chosen, are never a good measure for the column density. Both subthermal excitation and optical depth effects lower the integrated line emission relative to the value obtained by LTE projection as indicated by Padoan et al. (2000). Thus the deduction of column densities applying any $X$ factor will necessarily fail outside of a limited density range. In lines with low critical densities and low optical depths, like the lower ${ }^{13} \mathrm{CO}$ or $\mathrm{C}^{18} \mathrm{O}$ transitions, a constant $X$ factor, which is about ten times the LTE value, is found in a density range covering two orders of magnitude.
But this range is still limited, so that the derivation of column densities may easily fail even in this case. Insufficient spatial resolution may also mimic a constant $X$ factor by integrating over a range of factors given by the full distribution of column densities in a telescope beam. Optical depth effects compress the distribution of line intensities at large values and subthermal excitation stretches it at small values relative to the underlying column density distribution.

The lower transitions of CO isotopes always trace the largescale distribution of low-density gas. The spatial scaling behaviour of this translucent material is well recovered also from optically thick ${ }^{12} \mathrm{CO}$ lines. They fail, however, to detect any dense cores that may have formed from gravitational collapse. Nevertheless, it is possible to infer their existence from the measurement of different low- $J$ transitions due to their imprint on the global excitation structure in the clouds. Comparing intensity histograms, intensity scaling laws, and ripples in the line profiles of two different low- $J$ transitions observed with an excellent signal-to-noise ratio gives strong hints on the existence of dense structures on small scales. The differences are small for a large optical depth of the lines so that ${ }^{12} \mathrm{CO}$ observations are rarely useful. ${ }^{13} \mathrm{CO}$ or even $\mathrm{C}^{18} \mathrm{O}$ observations should be preferred. All significant deviations between the two lines indicate an inhomogeneous excitation pattern in the cloud which can be produced by gravitationally collapsed cores. We have to mention, however, that temperature variations, not considered in our cloud models, may lead to the same inhomogeneous excitation.

Only in high- $J$ transition the dense cores are directly visible. Their direct detection is, however, difficult as the emission is relatively weak, spatially strongly confined, and the location of the peak emission is not necessarily related to the peaks observed in any lower transition. The best way to trace the density structure is provided by dust observations, either in continuum emission or in dust extinction (Lada et al. 1994). Even the combination of several molecular transitions does in general provide less information on the column density structure. The molecular line observations are essential, however, to test any physical cloud model. Whereas numerous models fit the observed density scaling laws in molecular clouds (e.g. Mac Low \& Ossenkopf 2000; Ossenkopf et al. 2001b) it is the information from the velocity structure that helps to discriminate between them.

The probability distribution function of the velocity structure cannot be traced by the average line profile in any single transition. The wings of the distribution are enhanced in lines which are subthermally excited and suppressed in optically thick lines. Combining observations of both types may, however, reveal the true velocity PDF. In contrast the spatial scaling behaviour of the velocity structure is well traced by most molecular lines. Radiative transfer effects may only hide the physical dissipation limit in the measured velocity scaling.

\subsection{Cloud models}

The observed density scaling laws are only reproduced by hydrodynamic or magnetohydrodynamic models which are driven 
mainly on large scales, do not suffer from a long period of decay of the turbulent cascade, and have no strong magnetic fields parallel to the line of sight. The turbulence models considered here, however, fail to provide a good match to the line profiles which are typically observed in cold, quiescent molecular clouds. The internal structure of the line profiles at certain positions is too sparse in the models when compared with observations of cold clouds. But the line profiles help to constrain the turbulent velocity dispersion and the velocity scaling. The observed spectral index of the velocity scaling $\beta_{\text {vel }}$ of about four is consistent with the interpretation of a molecular cloud as shock dominated medium (Ossenkopf \& Mac Low 2002).

To overcome the problems of the turbulence models considered here, new models should include some treatment for the effective turbulence on a sub-resolution scale. Moreover, boundary effects have to be addressed. The density in the simulations should be adjusted to give a somewhat lower total mass compared to the values used here, as they represent at the moment only relatively dense parts of molecular clouds, not typical for the translucent medium on the scale of several parsecs. Future models should also include a self-consistent treatment of the energy balance in the turbulent structure. Here, a modified version of the radiative transfer code may help to take the cooling by molecular lines into account, but this requires a further speed up as the computation of the radiative transfer still needs considerably more time than a single step in the hydrodynamic or magnetohydrodynamic simulations. We have just finished one step in the systematic comparison of turbulence models with molecular cloud observations taking the radiative transfer into account. From the comparison we are already able to constrain essential parameters of molecular cloud turbulence. Using these results a new series of turbulence models should be set up to create finally a self-consistent physical picture of the turbulent structure in molecular clouds.

Altogether, the systematic application of the radiative transfer model for a wide range of molecular cloud simulations provides important insights into the relations between observed molecular line data and the underlying molecular cloud structures. It shows that great care is necessary in the analysis of molecular line observations and that it is essential to combine observations in different tracers to draw conclusions about the cloud structure. The comparison of different cloud models with observational data helps to discover basic parameters of the molecular cloud turbulence, but it shows also that we do not yet own a self-consistent picture of the structure formation in interstellar clouds explaining all observational facts.

Acknowledgements. I thank M.-M. Mac Low, R. Klessen, and F. Heitsch for providing me the data of their turbulence simulations and for many valuable debates and comments on this paper. Moreover, I want to thank J. Ballesteros-Paredes, F. Bensch, M.-M. Mac Low, and J. Stutzki for useful discussions. This work has been supported by the Deutsche Forschungsgemeinschaft through grants SFB 301C and 494B. It has made use of NASA's Astrophysics Data System Abstract Service.

\section{References}

Auer, L. H. 1987, in Numerical Radiative Transfer, ed. W. Kalkofen (Cambridge Univ. Press), 101

Ballesteros-Paredes, J., Vázquez-Semadeni, E., \& Scalo, J. 1999, ApJ, 515,286

Ballesteros-Paredes, J., Hartmann, L., \& Vázquez-Semadeni, E. 1999, ApJ, 527, 285

Bensch, F., Stutzki, J., \& Ossenkopf, V. 2001, A\&A, 366, 636

Le Bourlot, J. 1991, A\&A, 242, 235

Digel, S. W., Grenier, I. A., Heithausen, A., Hunter, S. D., \& Thaddeus, P. 1996, ApJ, 463, 609

Falgarone, E., \& Phillips, T. G. 1990, ApJ, 359, 344

Falgarone, E., Phillips, T. G., \& Walker, C. K. 1991, ApJ, 378, 186

Falgarone, E., Panis, J.-F., Heithausen, A., et al. 1998, A\&A, 331, 669

Flower, D. R., \& Launay, J. M. 1985, MNRAS, 214, 271

Goodman, A. A., Barranco, J. A., Wilner, D. J., \& Heyer, M. H. 1998, ApJ, 504, 223

Heithausen, A., \& Thaddeus, P. 1990, ApJ, 353, L49

Heithausen, A., Bensch, F., Stutzki, J., Falgarone, E., \& Panis, J. F. 1998, A\&A, 331, L65

Heitsch, F., Mac Low, M.-M., \& Klessen, R. S. 2001, ApJ, 547, 280

Juvela, M. 1997, A\&A, 322, 943

Klessen, R. S. 1997, MNRAS, 292, 11

Klessen, R. S. 2000, ApJ, 535, 869

Klessen, R. S., Heitsch, F., \& Mac Low, M.-M. 2000, ApJ, 535, 887

Kolmogorov, A. N. 1941, Dokl. Akad. Nauk SSSR, 30, 9

Kutner, M.-L., Rubio, M., Booth, R. S., et al. 1997, A\&AS, 122, 255

Lada, Ch. J., Lada, E. A., Clemens, D. P., \& Bally, J. 1994, ApJ, 429, 694

Larson, R. B. 1981, MNRAS, 194, 809

Mac Low, M.-M. 1999, ApJ, 524, 169

Mac Low, M.-M., \& Ossenkopf, V. 2000, A\&A, 353, 339

Mac Low, M.-M., Klessen, R. S., Burkert, A., \& Smith, M. D. 1998a, Phys. Rev. Lett., 80, 2754

Meyerdierks, H., \& Heithausen, A. 1996, A\&A, 313, 929

Miesch, M. S., \& Bally, J. 1994, ApJ, 429, 645

Mihalas, D. 1978, Stellar Atmospheres (W. H. Freeman and Co., San Francisco)

Müller, H. S. P., Thorwirth, S., Roth, D. A., \& Winnewisser, G. 2001, A\&A, 370, L49

Ossenkopf, V. 1997, New Astr., 2, 365

Ossenkopf, V., \& Mac Low, M.-M. 2002, A\&A, 390, 307

Ossenkopf, V., Bensch, F., \& Stutzki, J. 2000, in The Chaotic Universe, ed. V. G. Gurzadyan, \& R. Ruffini (World Sci.), 394

Ossenkopf, V., Trojan, Ch., \& Stutzki, J. 2001a, A\&A, 378, 608

Ossenkopf, V., Klessen, R., \& Heitsch, F. 2001b, A\&A, 379, 1005

Ostriker, E. C. 1999, in Interstellar Turbulence, ed. J. Franco, \& A. Carramiñana (Cambridge Univ. Press), 240

Padoan, P., Jones, J. T., \& Nordlund, A. P. 1997, ApJ, 474, 730

Padoan, P., Juvela, M., Bally, J., \& Nordlund, A. 1998, ApJ, 504, 300

Padoan, P., Bally, J., Billawala, Y., Juvela, M., \& Nordlund, Å. 1999, ApJ, 525, 318

Padoan, P., Juvela, M., Bally, J., \& Nordlund, Å. 2000, ApJ, 529, 259

Snell, R. L. 1981, ApJS, 45, 121

Sobolev, V. V. 1957, SvA, 1, 678

Stutzki, J., Bensch, F., Heithausen, A., Ossenkopf, V., \& Zielinsky, M. 1998, A\&A, 336, 697

Tauber, J. A. 1996, A\&A, 315, 591

Vázquez-Semadeni, E. 2000, in The Chaotic Universe, ed. V. G. Gurzadyan, \& R. Ruffini (World Sci.), 384

White, R. E. 1977, ApJ, 211, 744

Zielinsky, M., \& Stutzki, J. 1999, A\&A, 347, 633 